ARTICLE

DOI: $10.1038 /$ s41467-018-04759-8

\title{
UBXN3B positively regulates STING-mediated antiviral immune responses
}

\author{
Long Yang ${ }^{1,2}$, Leilei Wang ${ }^{1,3}$, Harshada Ketkar ${ }^{1}$, Jinzhu Ma,4, Guang Yang ${ }^{5}$, Shuang Cui ${ }^{6}$, Tingting Geng (D) 1 \\ Dana G. Mordue ${ }^{1}$, Toyoshi Fujimoto ${ }^{7}$, Gong Cheng ${ }^{8}$, Fuping You ${ }^{6}$, Rongtuan Lin², \\ Erol Fikrig 9,10 \& Penghua Wang ${ }^{1}$
}

The ubiquitin regulatory $X$ domain-containing proteins (UBXNs) are likely involved in diverse biological processes. Their physiological functions, however, remain largely unknown. Here we present physiological evidence that UBXN3B positively regulates stimulator-of-interferon genes (STING) signaling. We employ a tamoxifen-inducible Cre-LoxP approach to generate systemic Ubxn3b knockout in adult mice as the Ubxn3b-null mutation is embryonically lethal. Ubxn $3 b^{-/-}$, like Sting ${ }^{-/-}$mice, are highly susceptible to lethal herpes simplex virus 1 (HSV-1) and vesicular stomatitis virus (VSV) infection, which is correlated with deficient immune responses when compared to Ubxn36 $+/+$ littermates. HSV-1 and STING agonist-induced immune responses are also reduced in several mouse and human Ubxn $3 b^{-/-}$primary cells. Mechanistic studies demonstrate that UBXN3B interacts with both STING and its E3 ligase TRIM56, and facilitates STING ubiquitination, dimerization, trafficking, and consequent recruitment and phosphorylation of TBK1. These results provide physiological evidence that links the UBXN family with antiviral immune responses.

\footnotetext{
${ }^{1}$ Department of Microbiology and Immunology, New York Medical College, 15 Dana Road, Valhalla, NY 10595, USA. ${ }^{2}$ Lady Davis Institute-Jewish General Hospital, Department of Medicine, McGill University, 3755 Chemin de la Côte-Sainte-Catherine, Montreal, QC H3T 1E2, Canada. ${ }^{3}$ Department of Obstetrics and Gynecology, Shengjing Hospital, China Medical University, 110004 Shenyang City, Liaoning Province, China. ${ }^{4}$ College of Life Science and Technology, Heilongjiang Bayi Agricultural University, 163319 Daqing City, Heilongjiang Province, China. ${ }^{5}$ Department of Parasitology, School of Medicine, Jinan University, 510610 Guangzhou City, Guangdong Province, China. ${ }^{6}$ Beijing Key Laboratory of Tumor Systems Biology, Department of Immunology, School of Basic Medical Sciences, Institute of Systems Biomedicine, Peking University Health Science Center, 100083 Beijing, China. ${ }^{7}$ Department of Anatomy and Molecular Cell Biology, Nagoya University Graduate School of Medicine, 65 Tsurumai, Showa, Nagoya 466-8550, Japan. ${ }^{8}$ Tsinghua-Peking Center for Life Sciences, School of Medicine, Tsinghua University, 100084 Beijing, China. ${ }^{9}$ Section of Infectious Diseases, Yale University School of Medicine, 333 Cedar Street, New Haven, CT 06510, USA. ${ }^{10}$ Howard Hughes Medical Institute, 4000 Jones Bridge Road, Chevy Chase, MD 20815, USA. Correspondence and requests for materials should be addressed to L.Y. (email: longyangrich@hotmail.com) or to E.F. (email: erol.fikrig@yale.edu) or to P.W. (email: Penghua_wang@nymc.edu)
} 
T he mammalian innate immune system detects invading pathogens via several families of pattern recognition receptors, one of which the cyclic GMP-AMP synthase senses cytosolic DNA ${ }^{1}$ and synthesizes cGAMP ${ }^{2,3}$. cGAMP serves as a second messenger which binds an endoplasmic reticulum (ER) membrane adaptor, stimulator-of-interferon genes (STING), to trigger antiviral type I interferon (IFN-I) and inflammatory immune responses ${ }^{4-6}$. STING undergoes lysine K63-linked polyubiquitination mediated by E3 ligase tripartite motif containing 32 (TRIM32) and TRIM56 $6^{7,8}$, dimerizes ${ }^{7}$, and trafficks out of the ER to perinuclear vesicles ${ }^{6,9,10}$ where it recruits and activates the kinase TANK-binding kinase 1 (TBK1) and inhibitor of nuclear factor $\mathrm{\kappa B}$ kinase $\mathrm{s}^{5,11}$, and in turn innate immune responses. STING could also transmit signals from other DNA sensors such as DNA-dependent activator of IFN regulatory factors (IRFs), DEAD-Box helicase 41, DNA-dependent protein kinase, and IFN- $\gamma$-inducible protein 16; however, their function in IFN-I response is still controversial ${ }^{12}$. Being such a central adaptor in the DNA sensing pathway, STING signaling is stringently regulated by post-translational modifications. TBK1mediated phosphorylation of STING provides a "licensing" mechanism for TBK1 to phosphorylate IRF3 and induction of IFN-I ${ }^{13}$, while nucleotide-binding oligomerization domain receptor X1 sequesters STING to negatively regulate its signaling $^{14}$. K63-linked polyubiquitination by TRIM32, TRIM56, and mitochondrial E3 ubiquitin protein ligase 1 (MUL1), K27 polyubiquitination by ER-resident E3 ligase complex autocrine motility factor receptor (AMFR)-insulin-induced gene 1 (INSIG1) promotes STING signaling, $7,8,15$, while K48-linked polyubiquitination of STING by E3 ligases Ring finger protein 5 and TRIM30 $\alpha$ dampens its signaling by targeting it for proteasomal degradation ${ }^{16,17}$. Obviously, regulation of STING by ubiquitination is very complex and the in vivo physiological relevance of different types of ubiquitination and E3 ligases remains elusive.

The ubiquitin regulatory $\mathrm{X}$ (UBX) domain shares weak homology with ubiquitin at the protein level and adopts the same three-dimensional-fold as ubiquitin ${ }^{18}$. One of the best understood UBXN proteins, p47, is a cofactor of the AAA ATPase (ATPase associated with various cellular activities), $\mathrm{p} 97^{19}$. p97 is highly conserved across species and involved in diverse cellular processes, including ubiquitin-dependent protein degradation, vesicle fusion, and cell cycle ${ }^{18}$. Several UBXN proteins have been recently shown to bind p97 and a large number of E3 ubiquitin ligases $^{20}$, suggesting a potential role for UBXNs in regulation of global protein turnover and cellular signaling. However, the molecular function of the majority of UBXN proteins is largely unknown. Here we perform a family-based screening to identify a UBXN that could modulate STING-dependent signaling. Indeed, by utilizing dual luciferase reporter system, we identify that UBXN3B is a positive regulator of STING and that UBXN3B complexes with STING and TRIM56 to potentiate STINGdependent innate immune responses.

\section{Results}

UBXN3B regulates STING-dependent IFN-I induction. We performed an IFN-stimulated response element (ISRE) reporter activation screening in human embryonic kidney 293 cells transformed with T antigen of SV40 (HEK293T) cells for UBXNs that enhanced a STING-mediated IFN response. Transfection with HA-STING plasmid and vector induced ISRE activity by $\sim 150$-fold relative to vector alone (Supplementary Fig. 1a). Interestingly, among all the UBXNs tested, UBXN3B most significantly potentiated a STING-induced IFN-I response, which was $\sim 5$-fold higher than STING alone (Supplementary Fig. 1a).
Overexpression of UBXN3B alone did not induce ISRE significantly (Supplementary Fig. 1b-d), indicating that UBXN3B is a positive regulator of STING-dependent signaling. To validate the screening results, we used cGAMP, a second messenger that binds and stimulates STING signaling ${ }^{1,21}$. cGAMP was used to prime HEK293T cells stably expressing FLAG-STING (designated HEK293T-STING cell line) following transfection with a UBXN3B expression plasmid. We found that UBXN3B synergized with cGAMP to induce an IFN-I response in a dosedependent manner (Supplementary Fig. $1 \mathrm{~b}$ and 1c). To confirm that the enhancing effect on STING signaling was specific to $\mathrm{UBXN} 3 \mathrm{~B}$, we included two UBXN members, UBXN3A which is the closest sibling of UBXN3B and UBXN9 which is more dissimilar from UBXN3B, in terms of domain structure ${ }^{20}$. Indeed, only UBXN3B, but not UBXN3A or UBXN9, enhanced the cGAMP-induced IFN-I response (Supplementary Fig. 1d). In addition, we tested if UBXN3B has a modulatory effect on other important antiviral signaling pathways. The results demonstrate that UBXN3B failed to synergize with retinoic acid-inducible gene I (RIG-I)-like receptor (RLR)-mediated and Toll-like receptor (TLR)-mediated IFN-I induction (Supplementary Fig. 2). These data suggest that UBXN3B specifically targets STING signaling.

UBXN3B is critical for anti-DNA virus immune responses. UBXN3B, also known as Fas-associated factor 2 and UBXD8, might play a role in regulating lipid metabolism ${ }^{22-24}$. However, its physiological function remains largely unclear. We therefore attempted to generate general $U b \times n 3 b^{-1-}$ mice using the CRISPR-Cas9 technology and unfortunately failed, suggesting that Ubxn3b deletion is embryonically lethal, consistent with a recent publication ${ }^{24}$. We therefore planned to generate an inducible conditional knockout strain so as to systemically ablate UBXN3B gene expression. We obtained $U b x n 3 b^{\text {flox/flox }}$ (exon 1, homologous recombination) from our collaborator ${ }^{24}$ and crossed it with a tamoxifen-inducible Cre mouse line $\mathrm{e}^{25,26}$, to generate a $\mathrm{Cre}^{+l-} U b x n 3 b^{\text {flox/flox }}$ mouse. The tamoxifen-inducible Cre model has been successfully applied to many gene deletions including essential genes ${ }^{25}$. Indeed, Ubxn3b protein expression was abolished in various tissues of tamoxifen-treated Cre $+/-U b x n 3 b^{\text {flox/flox }}$ mice (Fig. 1a). However, no discernible developmental defects or behavioral abnormality were observed in these mice. We then infected tamoxifen-pretreated $\mathrm{Cre}^{+/-}$ $U b x n 3 b^{\text {flox/flox }}$ (referred to as $U b \times n 3 b^{-1-}$ for convenience throughout the manuscript) or untreated $\mathrm{Cre}^{+} U b \times n 3 b^{\text {flox/flox }}$ (referred to as $U b \times n 3 b^{+/+}$) littermates with a DNA virus, herpes simplex virus 1 (HSV-1), which effectively induces STING signaling. To exclude any potential side effects of tamoxifen on HSV-1 pathogenesis and/or antiviral immunity, we included tamoxifen-treated $\mathrm{Cre}^{+/-}$and $U b \times n 3 b^{\text {flox/flox }}$ littermates as controls. As shown in Fig.1b, $U b \times n 3 b^{-l-}$ mice $(N=8)$ died of HSV1 infection earlier than $U b \times n 3 b^{+/+}$mice $(N=8)$ and the other control mice $(N=9)$. The overall survival rate of $U b \times n 3 b^{-1-}$ mice was also much lower $(P<0.01, \log$-rank test). In addition, all infected $U b \times n 3 b^{-1-}$ mice developed severe neurological symptoms, as well as hunched posture, decreased movement, and labored breathing on day 3 after infection. These symptoms were completely absent from $U b \times n 3 b^{+/+}$animals (Supplementary Movie 1). Compared to Sting ${ }^{-1-}$ mice $(N=8), U b \times n 3 b^{-l-}$ mice $(\mathrm{N}=9)$ were a bit less sensitive to HSV-1 infection (Fig. 1c). In agreement with the survival data, the serum IFN-I abundance was markedly decreased at 4 and $8 \mathrm{~h}$ after HSV-1 inoculation in $U b \times n 3 b^{-I-}(N=6)$ compared to Ubxn $3 b^{+/+}(N=10)$ mice (non-parametric Mann-Whitney test, $P<0.05$ ), demonstrating that UBXN3B is critical for HSV-1-induced IFN response (Fig. 1d). In agreement with this, the mRNA levels of Ifnb1, Tnfa, 

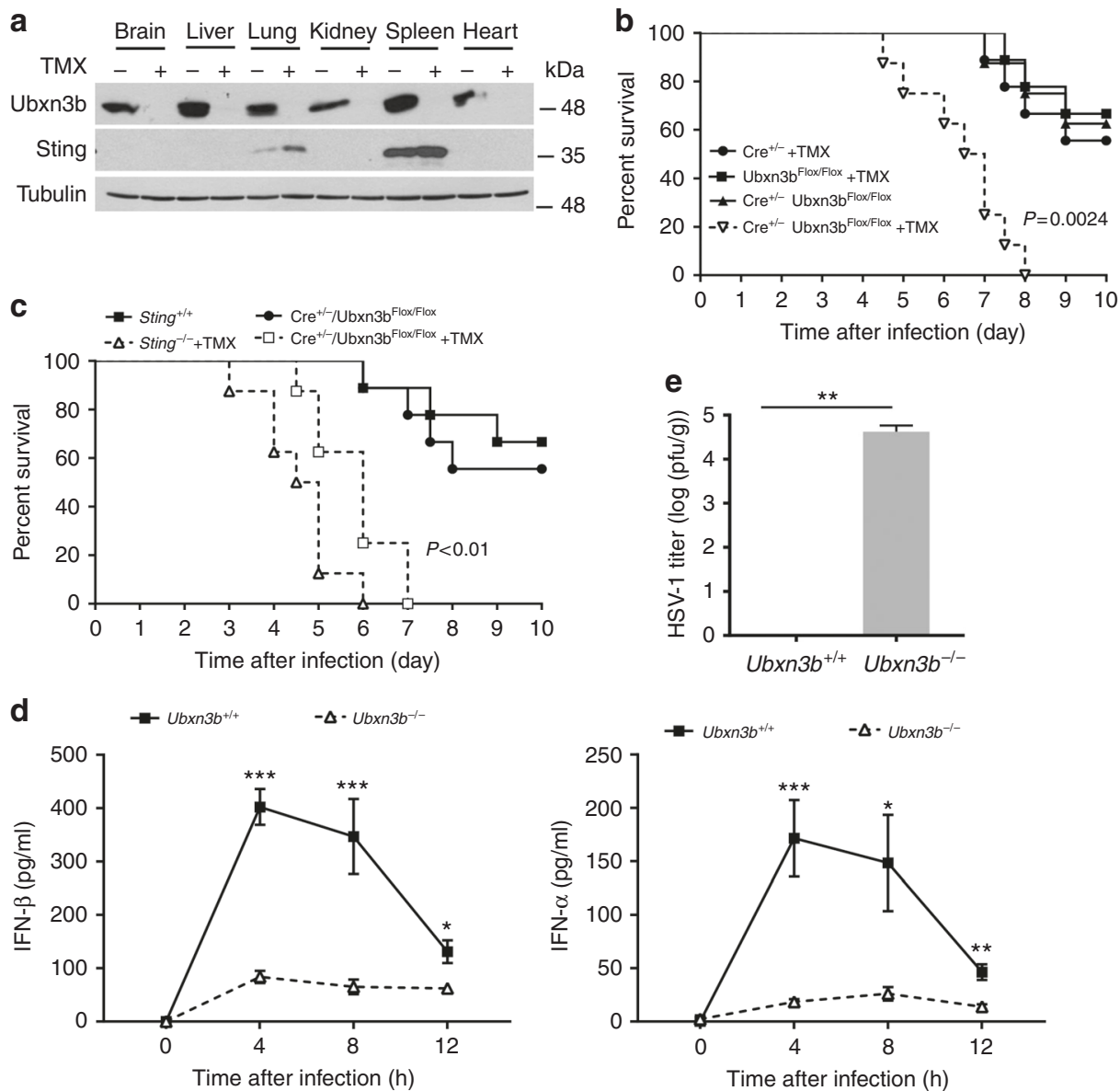

Fig. 1 UBXN3B is critical for IFN-I induction by and control of HSV-1 infection in vivo. a Immunoblots showing Ubxn3b knockout efficiency in various tissues without/with tamoxifen (TMX) treatment in $\mathrm{Cre}^{+/-} \mathrm{Ubxn} 3 b^{\text {flox/flox }}$ mice. Tubulin is a housekeeping protein control. b, c The survival curves of tamoxifen

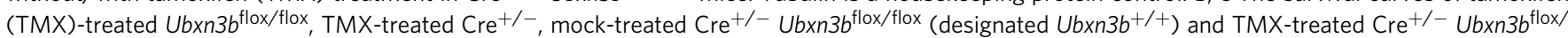
flox (designated Ubxn3b-/-) littermate and Sting ${ }^{-/-}$mice challenged with $1 \times 10^{7}$ plaque-forming units (PFU) per mouse of HSV-1 i.v. $N=8-9$ mice/ genotype. ${ }^{\star \star \star} P<0.001$ (log-rank test). d The serum IFN-I concentrations (mean \pm s.e.m) of mice challenged with $2 \times 10^{6} \mathrm{PFU}$ per mouse of HSV-1 i.v. ${ }^{\star \star} P<$ 0.01 ; ${ }^{\star} P<0.05$ (non-parametric Mann-Whitney test), $N=10\left(U b \times n 3 b^{+/+}\right)$or $N=6\left(U b \times n 3 b^{-/-}\right)$. e The viral titers (mean \pm s.e.m) in the brain on day 3 after infection (PFU per gram tissue). ${ }^{\star \star} P<0.01$ (non-parametric Mann-Whitney test), $N=5$ mice per genotype. The data are pooled from two independent experiments

and Ifit1 (an IFN-I-induced antiviral effector) in leukocytes of $U b \times n 3 b^{-1-}$ mice were also lower than those in $U b \times n 3 b^{+1+}$ littermates $(N=5$ each genotype, $P<0.05$, non-parametric Mann-Whitney test) (Supplementary Fig. 3). Given that HSV-1 can penetrate the brain and elicit fatal encephalitis in mice, we then quantified HSV-1 titers in the brain by plaque-forming assay. Ubxn $3 b^{-1-}$ mice had a significantly increased number of viral particles compared to Ubxn $3 b^{+1+}$ mice on day 3 after infection $(N=5$ each genotype, $P<0.01$, non-parametric Mann-Whitney test) (Fig. 1e).

We next asked if the antiviral function of UBXN3B was celltype specific. We isolated bone marrow cells from $\mathrm{Cre}^{+l-}$ $U b \times n 3 b^{\text {flox/flox }}$ mice pretreated without $\left(U b \times n 3 b^{+/+}\right)$or with tamoxifen (Ubxn3b- $b^{-}$) and differentiated them into macrophages (bone marrow-derived macrophage (BMDMs)) with L929 cell-conditioned medium, conventional dendritic cells (cDCs) with granulocyte-macrophage colony-stimulating factor (GM$\mathrm{CSF}$ ) and plasmacytoid DC (pDCs) with Flt3L. Induction of IFNI protein by HSV-1, STING-stimulating immunostimulatory DNA (ISD) and cGAMP was remarkably compromised in knockout cells (Fig. 2a). Correspondingly, induction of Oas1a protein, an IFN-stimulated gene (ISG), was also impaired in knockout cDC cells (Fig. 2b). The mRNA levels of Ifnbl and Tnfa were decreased in $U b x n 3 b^{-1-}$ cells at $6 \mathrm{~h}$ after infection, which was accompanied by increased viral load (Fig. 2c, d). The abovementioned immune cells are not very permissive to HSV-1 Infection. We next isolated mouse embryonic fibroblasts (MEFs), which are highly permissive to HSV-1 in contrast to the above-mentioned immune cells we induced in vitro, from untreated $\mathrm{Cre}^{+l-} U b \times n 3 b^{\text {flox/flox }} \mathrm{E} 14$ embryos and induced Ubxn3b deletion with 4-hydroxyl tamoxifen in vitro. Ubxn3b protein expression was almost completely depleted after 4-hydroxyl tamoxifen treatment in $\mathrm{Cre}^{+l-} U b \times n 3 b^{\text {flox/flox }}$ $\left(U b \times n 3 b^{-I-}\right)$ cells (Fig. 2e). In agreement with the aforementioned results, the viral titers produced by $U b \times n 3 b^{-1-}$ cells were higher than $U b x n 3 b^{+/+}$(Fig. 2f). Ifnb1 and its ISG mRNA levels induced by HSV-1 were consistently down-regulated in Ubxn $3 b^{-l-}$ cells (Fig. 2g).

The UBXN3B protein is evolutionarily conserved, with $97 \%$ identity between human and rodent. We then asked if its antiviral function is also evolutionarily conserved. We employed the CRISPR-Cas9 technology to generate $U B X N 3 B^{-1-}$ in H1975 cell, a human lung epithelial cell line (Supplementary Fig. 4a). Consistent with the results from mouse cells, IFNB1 induction by ISD was much lower in $U B X N 3 B^{-1-}$ cells than wild type (WT) cells. But the dsRNA analog polyinosinic:polycytidylic acid 
(polyIC)-induced IFNB1 expression was not significantly impaired (Supplementary Fig. 4b). The HSV-1 titers (Supplementary Fig. 4c) and intracellular HSV-1 protein levels (Supplementary Fig. $4 \mathrm{~d}$ ) were very much increased in $U B X N 3 B^{-/-}$cells compared to WT cells, suggesting a critical antiviral role for
UBXN3B. The immune responses to HSV-1 including IFN-I (IFNB1) and inflammatory response (TNFA) were reduced in the absence of UBXN3B expression (Supplementary Fig. 4e). To extend this further, we next examined the UBXN3B antiviral function in primary human trophoblasts. These cells (1) may be
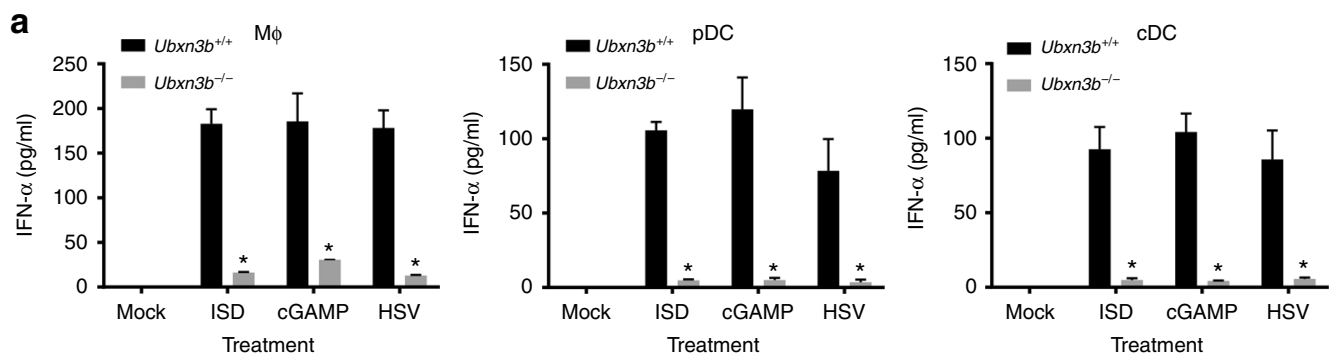

b
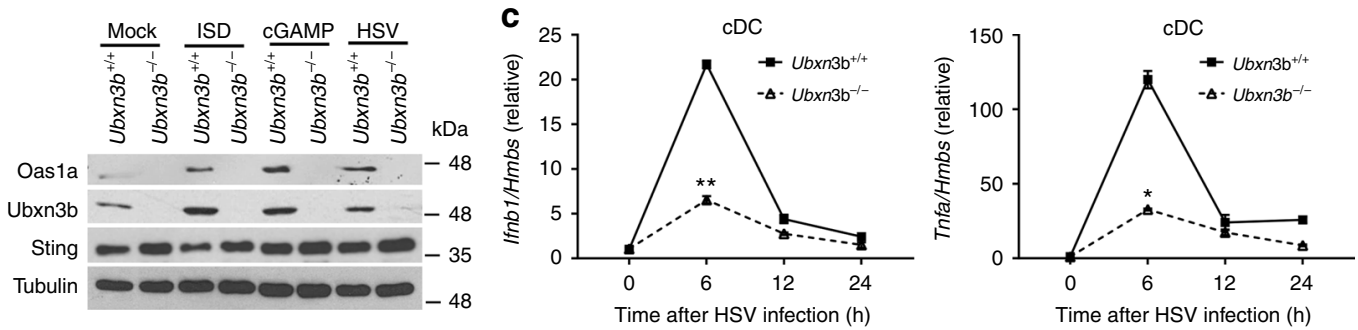

d

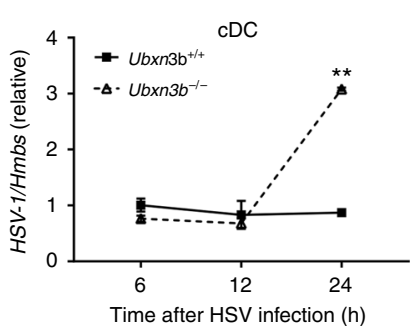

e
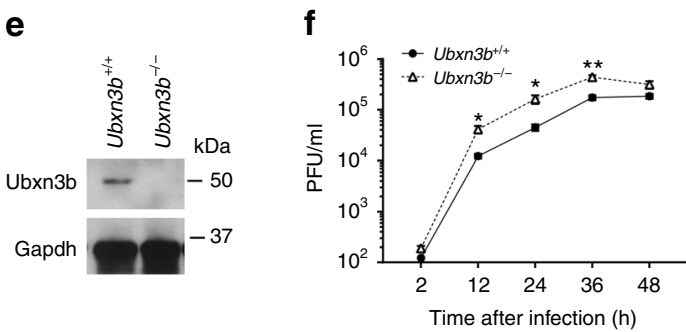

$\mathbf{g}$
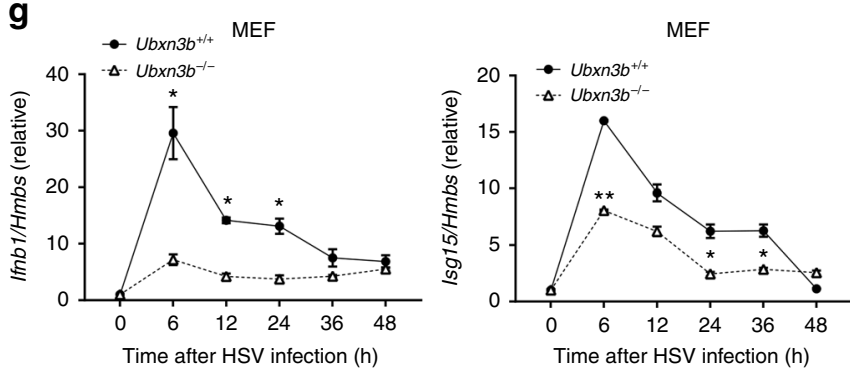

h
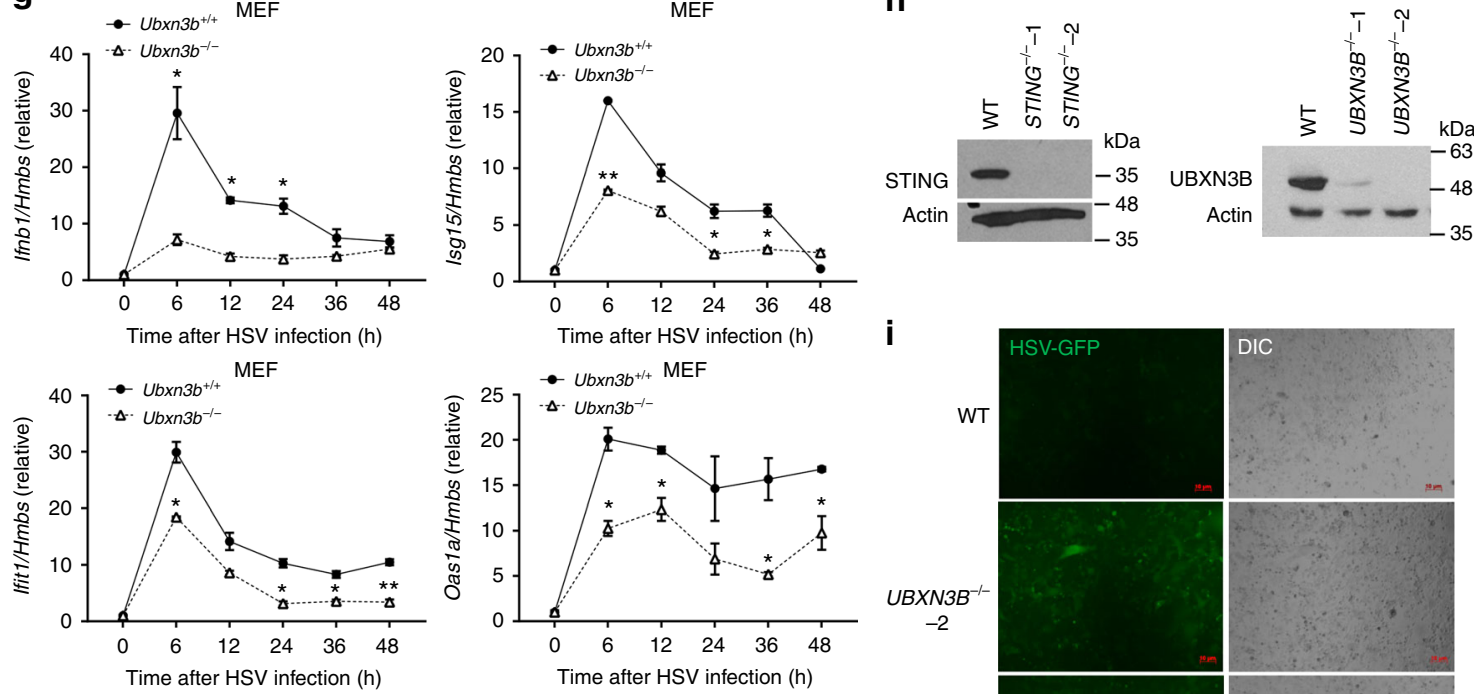

i

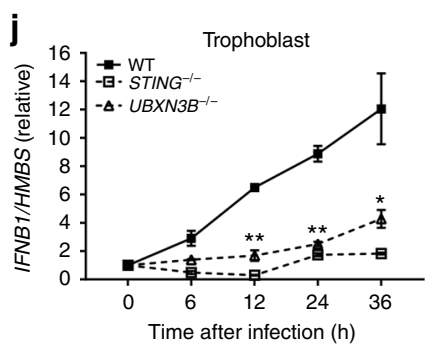



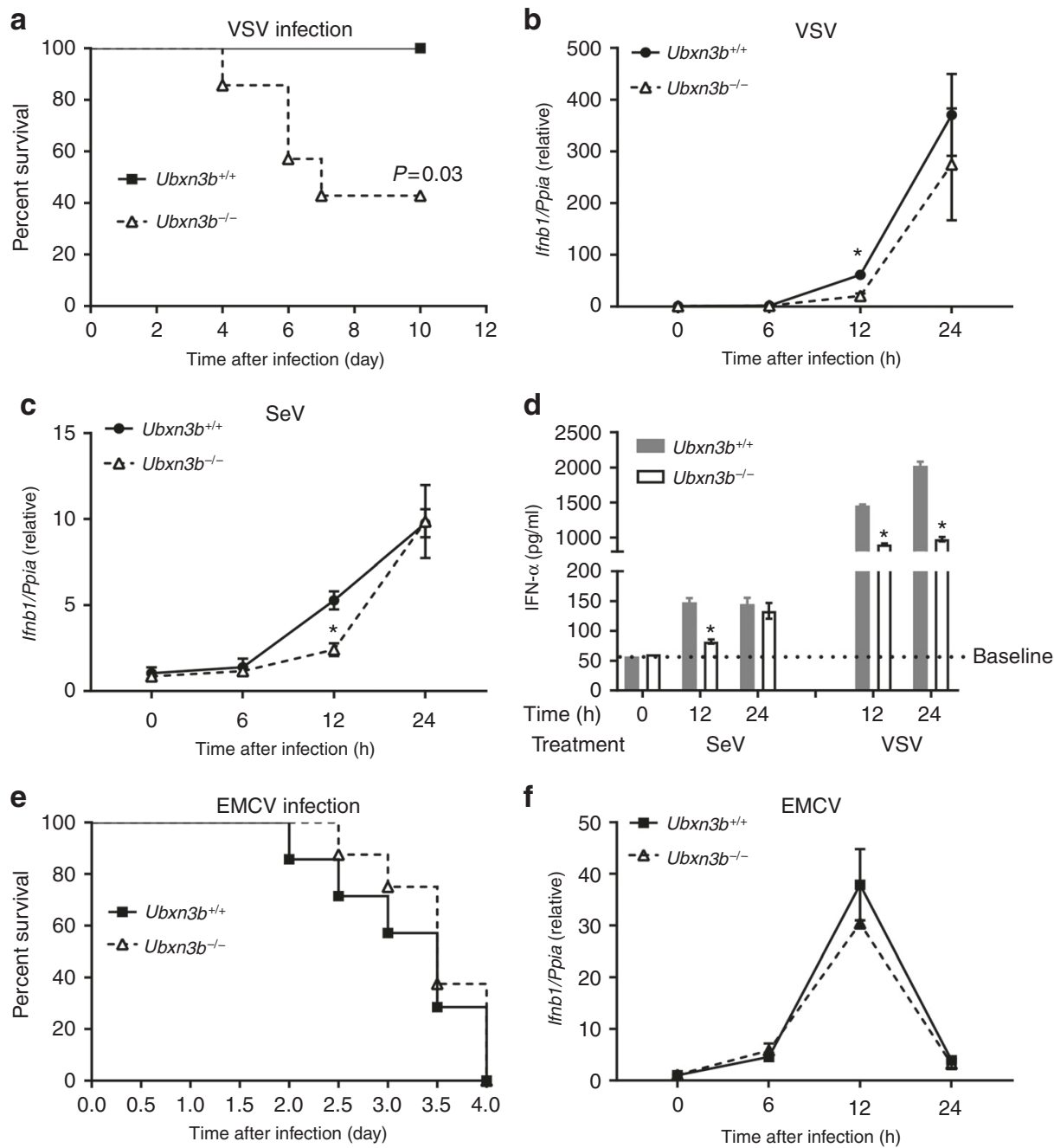

Fig. 3 UBXN3B is critical for type I IFN responses to SeV and VSV, but not EMCV infection. a The survival curves of Ubx3b-/- $(N=6)$ and Ubxn3b $b^{-/-}(N$ $=7$ ) mice infected with $1 \times 10^{7} \mathrm{PFU}$ of VSV i.v. The results are pooled from two experiments. ${ }^{\star} P<0.05$ (log-rank test). b, c qPCR analysis of Ifnb1 expression in cDCs infected with $\mathbf{b}$ VSV $(\mathrm{MOI}=5)$ or $\mathbf{c} \mathrm{SeV}$ (200 hemagglutination units per $10^{5}$ cells). d ELISA of IFN- $\alpha$ in the cell culture supernatants of cDCs infected with SeV and VSV as in b, c. $N=3$ per genotype per time point. ${ }^{\star} P<0.05$ (unpaired Student's $t$ test). e The survival curves of Ubx3b ${ }^{+/+}$( $N$ $=7)$ and $U b x n 3 b^{-/-}(N=8)$ mice infected with 200 PFU of EMCV i.p. $P=0.53$ (log-rank test). The results are pooled from two experiments. $\mathbf{f}$ qPCR analysis of Ifnb1 expression in $\mathrm{CDC}$ infected with EMCV $(\mathrm{MOI}=5)$ for the indicated time. Bars/data points: mean \pm s.e.m. Two biological replicates were pooled for $\mathrm{qPCR}\left(N=2\right.$ per genotype per time point). ${ }^{\star} P<0.05$ (unpaired Student's $t$ test). The results are representative of two independent experiments

physiologically related to vertical transmission of HSV-1; (2) can be passaged in vitro for up to $12-15$ generations $^{27}$, which allows us to knockout the genes of interest with CRISPR-Cas9 and examine their functions; and (3) can be obtained in a large quantity. In parallel, we included STING ${ }^{-1-}$ as a positive control. As shown in Fig. 2h, STING and UBXN3B protein expression was depleted in knockout cells. Both STING ${ }^{-1-}$ and $U B X N 3 B^{-1-}$ cells were more permissive to HSV-1 infection than WT (Fig. 2i); the

Fig. 2 UBXN3B is critical for STING-dependent IFN-I induction in mouse primary cells. a ELISA of IFN- $\alpha$ in the cell culture supernatants of (Ubxn3b $b^{+/+}$,

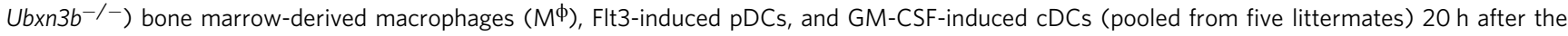
indicated treatments. $N=3$ per genotype. ${ }^{\star} P<0.05$ (unpaired Student's $t$ test). $\mathbf{b}$ Immunoblots of an interferon-stimulated gene Oas1a, Sting, and Ubxn $3 b$ expression in CDCs $20 \mathrm{~h}$ after the indicated treatments. Tubulin is a housekeeping protein control. qPCR analysis of (c) Ifnb1 and Tnfa mRNA expression and $\mathbf{d}$ cellular HSV-1 genome loads in cDCs infected with HSV-1 $(\mathrm{MOI}=10)$ for the indicated time. e Immunoblots showing Ubxn3b and housekeeping Gapdh protein expression in mock (Ubxn $3 b^{+/+}$) or 4-hydroxyl tamoxifen-treated Cre ${ }^{+/-}$Ubxn3b flox/flox (Ubxn3b-/-) MEFs. $\mathbf{f}$ Viral titers (plaque-forming units $/ \mathrm{ml}$ ) in the supernatant of MEFs infected with HSV-1 $(\mathrm{MOI}=0.1) . N=3$ per genotype per time point. ${ }^{\star} P<0.05$; ${ }^{\star \star} P<0.01$ (unpaired Student's $t$ test). $\mathbf{g}$ qPCR analysis of selected immune gene mRNA expression in MEFs infected with HSV-1 as in $\mathbf{g}$. $\mathbf{h}$ The immunoblots show knockout efficacy of STING and UBXN3B by CRISPR-Cas9 in human primary trophoblasts. Actin is a housekeeping protein control. i Fluorescent microscopic images of human primary trophoblasts infected with HSV-1-GFP $(\mathrm{MOI}=0.3)$ for $18 \mathrm{~h}$. Objective, $\times 5$. Scale bar, $10 \mu \mathrm{m}$. $\mathbf{j}$ qPCR analysis of Ifnb1 mRNA expression in human primary trophoblasts infected with HSV-1-GFP for the indicated time. Bars/data points: mean \pm s.e.m. Two biological replicates were pooled for qPCR $(N=2$ per genotype per time point). ${ }^{\star} P<0.05$; ${ }^{\star \star} P<0.01$ (unpaired Student's $t$ test). The results are representative of two independent experiments 
IFNB1 mRNA expression was, however, much lower in knockout cells (Fig. 2j). These results demonstrate that the STINGregulating function of UBXN3B is evolutionarily conserved.

UBXN3B regulates immune responses to some RNA viruses. STING signaling is not only essential for induction of immune responses to DNA viruses but also important for antiviral immunity to certain RNA viruses such as VSV and Sendai virus $(\mathrm{SeV})$. We then investigated the physiological role of UBXN3B during RNA virus infection. Consistent with the phenotype of Sting $^{-1-}$ infected with $\mathrm{VSV}^{5}, \operatorname{Ubxn} 3 b^{-1-}$ mice $(N=6)$ were also more susceptible to lethal VSV infection than its $U b \times n 3 b^{+/+}$ littermates $(N=7$ each genotype, $P<0.05$, log-rank test) (Fig. 3a). The Ifnb1 mRNA expression was modestly decreased in knockout cDCs at $12 \mathrm{~h}$ after infection (Fig. 3b). Similarly the Ifnb1 transcripts were also reduced in knockout cells infected with $\mathrm{SeV}$ (Fig. 3c). Consistent with this, the IFN- $\alpha$ protein concentration in the knockout cell supernatants was modestly lower than WT (Fig. 3d). However, Ubxn3b was dispensable for the control of a non-enveloped $(+)$ single-stranded RNA virus, encephalomyocarditis virus (EMCV) infection in vivo (Fig. 3e), and for innate immune responses in cDCs (Fig. 3f). This is also consistent with the phenotype of Sting ${ }^{-1-}$ mice $^{5}$.

UBXN3B regulates STING signaling. All the aforementioned in vivo and in vitro results clearly indicate an essential role of UBXN3B in the STING signaling pathway. To see whether UBXN3B also plays a role in other pathogen pattern recognition receptors such as RLR and TLR signaling pathways, we examined immune response induction in CDCs by several well-established
RLR/TLR agonists. The results show that Ifnb1 and Tnfa mRNA upregulation by CpG DNA (TLR9), FLS-1 (Pam2CGDPKHPKSF, TLR2/6), lipopolysaccharide (LPS, TLR4), high-molecular-weight polyIC (TLR3, MDA5), and single-stranded poly-uridine (polyU, TLR7) in Ubxn3 $3 b^{-1-}$ was not significantly different from Ubxn3b $+I+$ cDCs (Supplementary Fig. 5a-e). These data demonstrate that UBXN3B is not essential for TLR/RLR-dependent signaling $^{28}$. We next asked if UBXN3B regulated the IFN-JAK-STAT pathway, which induces expression of a large number of antiviral effectors. As shown in Supplementary Fig. 5f, the Isg15 and Oas1a mRNA expression levels were similar between $U b \times n 3 b^{+/+}$and $U b \times n 3 b^{-1-}$ CDCs, suggesting that UBXN3B is dispensable for the JAK-STAT signaling pathway. Furthermore, we observed that $U b \times n 3 b$ expression was induced by IFN- $\alpha$ in both $\mathrm{cDCs}$ and MEFs, which was completely dependent on the IFN-I receptor signaling (Supplementary Fig. 6). Intriguingly, we noted that $U b \times n 3 b$ was gradually and constantly upregulated throughout IFN- $\alpha$ treatment (Supplementary Fig. 6a). These data suggest that UBXN3B is an ISG.

We next explored the molecular mechanism by which UBXN3B acts on STING-dependent signaling. Lysine (K) 63linked ubiquitination and dimerization of STING is a prerequisite for STING trafficking out of the ER to perinuclear vesicles where it recruits the antiviral kinase TBK1 to induce IFN-I $\mathrm{I}^{7,9,10,29}$. STING is then phosphorylated and degraded via autophagy ${ }^{29}$ and proteasomes $^{30}$. UBXN proteins are likely involved in regulation of E3 ubiquitin ligases ${ }^{20}$. We first asked whether UBXN3B played a role in STING dimerization, trafficking, and phosphorylation. Indeed, STING dimerization took places $8 \mathrm{~h}$ after HSV-1 infection in $U b \times n 3 b^{+1+}$ MEFs, but this was obviously reduced in Ubxn $3 b^{-1-}$ cells (Fig. 4a). We observed a similar phenotype in

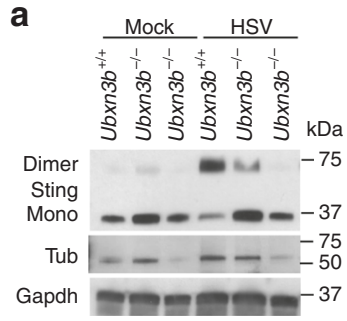

b

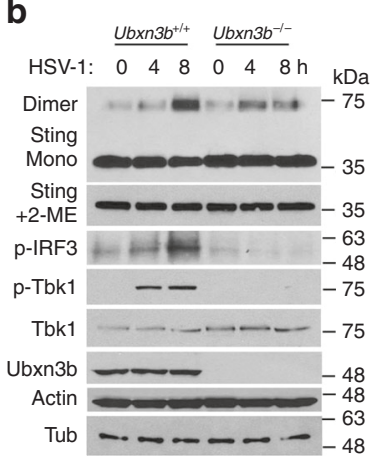

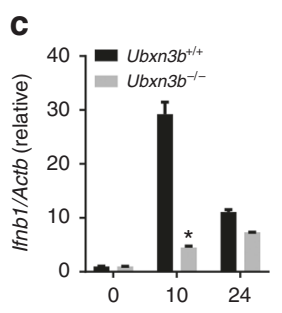

Time after infection (h)

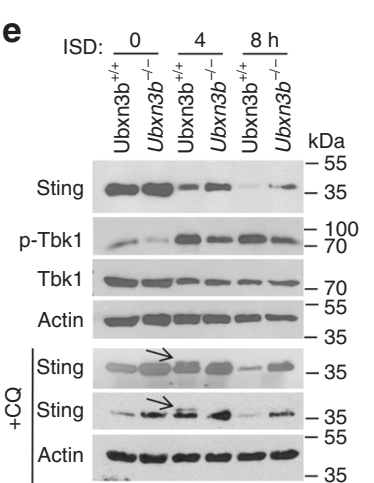

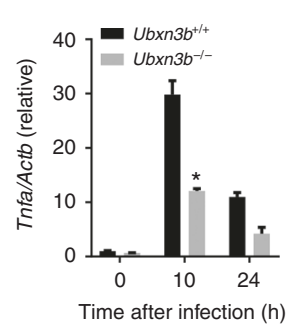
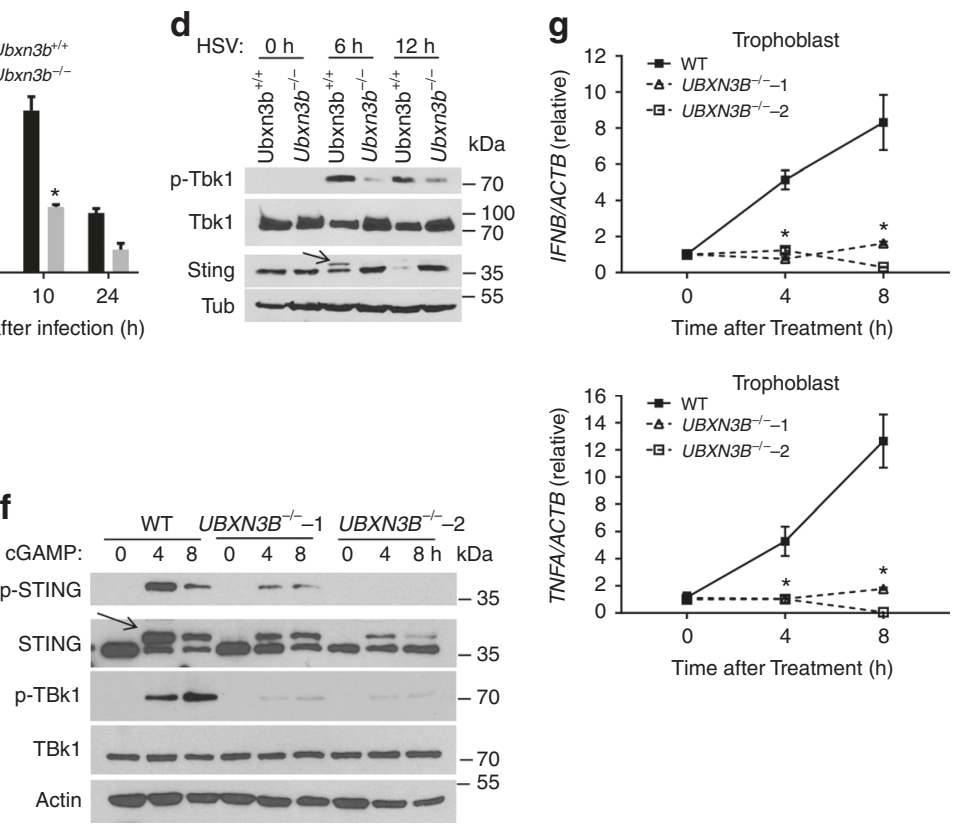
.

Fig. 4 UBXN3B regulates STING dimerization, phosphorylation, and degradation. a Immunoblots showing Sting dimerization in untreated $\left(U b x n 3 b^{+/+}\right)$and 4-hydroxyl tamoxifen-induced $\mathrm{Cre}^{+/-} \mathrm{Ubxn} 3 \mathrm{~b}^{\text {flox/flox }}\left(\mathrm{Ubxn3b^{-/- }}\right)$ primary MEFs. The cells were infected without (mock) or with HSV-1 (MOI = 0.5) for $8 \mathrm{~h}$. b Immunoblotting analysis of the whole-cell lysates of bone marrow-derived cDCs infected with HSV-1 (MOI=5). Mono monomer, 2-ME $\beta$-mercaptoethanol, a chemical compound that reduces disulfide bonds. c qPCR quantification of Ifnb1 and Tnfa mRNA induction in cDCs by HSV-1 $(\mathrm{MOI}=5)$. Bars: mean \pm s.e.m. Two biological replicates were pooled for qPCR ( $N=2$ per genotype per time point). ${ }^{\star} P<0.05$ (unpaired Student's $t$ test). d-f Immunoblotting analysis of the whole-cell lysates of $\mathbf{d}$ MEFs infected with HSV-1 (MOI $=0.5)$, e MEFs transfected with ISD ( $8 \mu \mathrm{g} / \mathrm{ml})$ in the absence or presence of $40 \mu \mathrm{M}$ of chloroquine ( $+\mathrm{CQ})$ and $\mathbf{f}$ trophoblasts transfected with cGAMP $(8 \mu \mathrm{g} / \mathrm{ml})$. In $\mathbf{e}$, $\mathbf{f}$, the arrows indicate phosphorylated STING with long and short exposure. Actin, Tubulin (Tub), and GAPDH are housekeeping protein controls. $\mathbf{g}$ qPCR quantification of IFNB1 and TNFA mRNA induction in trophoblasts transfected with cGAMP $(8 \mu \mathrm{g} / \mathrm{ml})$. In $\mathbf{c}, \mathbf{g}$, the bars are: mean \pm s.e.m. Two biological replicates were pooled for qPCR $(N=2$ per genotype per time point). ${ }^{\star} P<0.05$ (unpaired Student's $t$ test). The results are representative of two to three independent experiments 
cDCs (Fig. 4b). Moreover, HSV-1-induced phosphorylation of TBK1 and IRF3 was inhibited in Ubxn3 $3 b^{-1-}$ cDCs (Fig. 4b). Consequently, Ifnb1 and Tnfa mRNA transcripts were also decreased in Ubxn $3 b^{-1-}$ (Fig. 4c). STING is degraded after HSV1 infection in permissive cells like MEFs. We extended the HSV-1 infection and indeed observed overt Sting degradation in Ubxn $3 b$ $+/+$ cells at $12 \mathrm{~h}$ and this was inhibited in knockout cells (Fig. $4 \mathrm{~d}$ ). A similar result was noted in H1975 cells (Supplementary Fig. 7). ISD also stimulated STING degradation, which was partially blocked in Ubxn $3 b^{-1-}$ (Fig. 4e). These data suggest that STING translocation from the ER to perinuclear vesicles is influenced by UBXN3B deficiency too. We investigated this in H1975 cells by immunofluorescence microscopy. In unstimulated cells, STING co-localized with an ER-resident protein calreticulin very well. STING was localized to some punctate structures that were away from calreticulin-stained ER $3 \mathrm{~h}$ after ISD stimulation in WT cells, but remained co-localized with calreticulin in ISDtreated $U B X N 3 B^{-1-}$ cells (Supplementary Fig. 8). Following translocation, STING is phosphorylated and degraded partially by autophagy. We did not readily detect phospho-Sting in ISDtreated MEFs, likely because of its rapid degradation following phosphorylation in these cells. We thus added chloroquine to block autophagy-mediated Sting degradation in order to capture
phospho-Sting. Indeed, chloroquine treatment delayed Sting degradation and Sting phosphorylation was observed at $4 \mathrm{~h}$ after ISD treatment in $U b \times n 3 b^{+/+}$cells but clearly absent in $U b \times n 3 b^{-l-}$ MEFs (Fig. 4e). Interestingly, STING phosphorylation was much readily detected in human trophoblasts. Using a specific antibody against only the phosphorylated human STING, we noted rapid phosphorylation of STING at $4 \mathrm{~h}$ after cGAMP treatment. STING phosphorylation was obviously reduced, so was TBK1; while STING degradation was inhibited in $U B X N 3 B^{-/-}$cells (Fig. 4f). As a result, IFNB1 and TNFA mRNA upregulation by cGAMP was repressed in $U B X N 3 B^{-1-}$ cells (Fig. $4 \mathrm{~g}$ ).

The aforementioned data clearly demonstrate that UBXN3B regulates STING signaling and its downstream immune responses. To test if UBXN3B directly participated in STING signaling complex, we employed a co-immunoprecipitation assay in HEK293T cells with simultaneous expression of FLAG-tagged UBXNs and HA-tagged STING. UBXN3B but not its sibling UBXN3A co-immunoprecipitated with STING (Fig. 5a), suggesting that UBXN3B-STING binding is specific and that UBXN3B is involved in STING signaling. By fluorescence microscopy, we observed that UBXN3B co-localized with STING to the ER in unstimulated cells (Mock), and partly translocated together with STING to punctate structures after ISD treatment (Fig. 5b). We
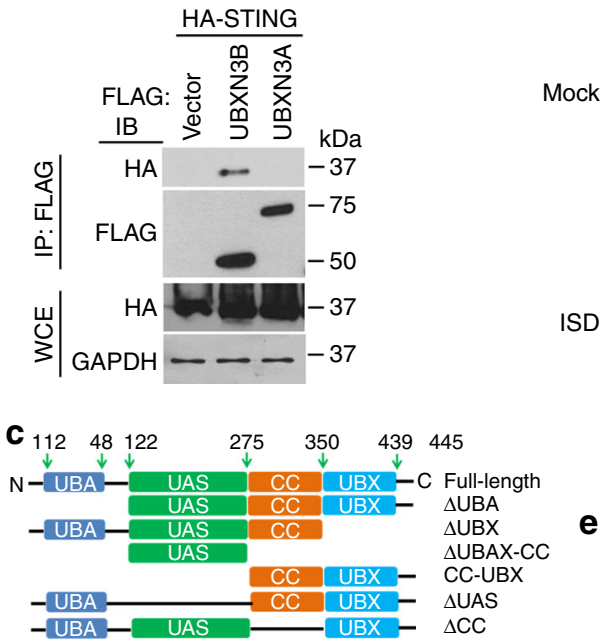

d

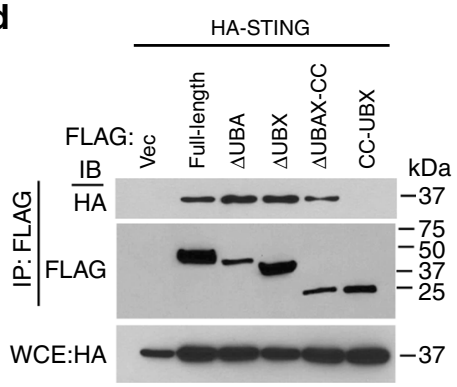

b
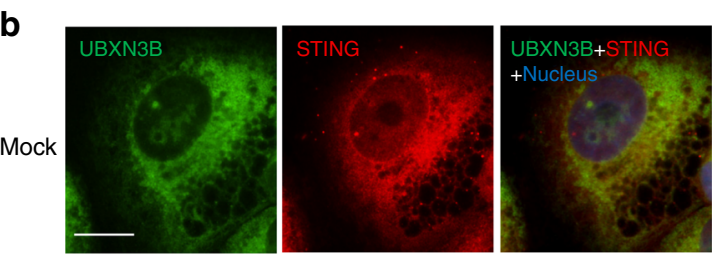

ISD
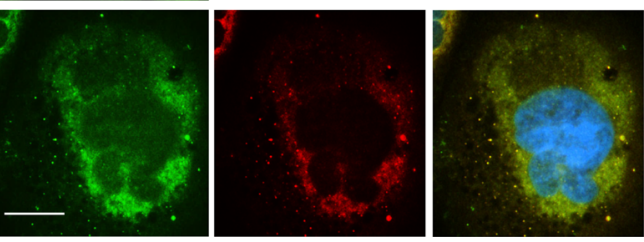
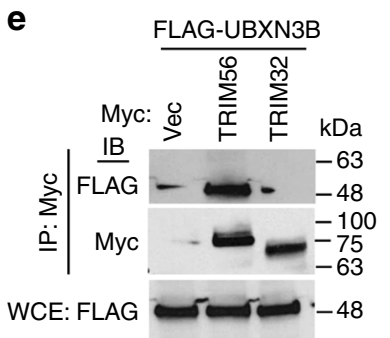

f

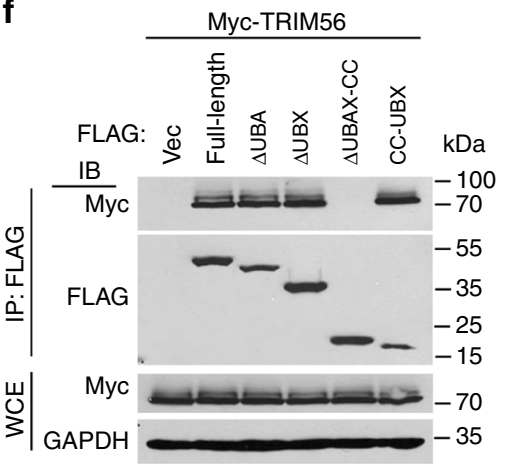

Fig. 5 UBXN3B interacts with STING and TRIM56. a Co-immunoprecipitation (co-IP) of STING with UBXN3B from HEK293 cells transfected with FLAG-UBXNs and HA-STING plasmids using anti-FLAG magnetic beads, followed by immunoblotting (IB) with an anti-HA (STING) and FLAG (UBXN) antibody. b Immunofluorescence staining of STING and UBXN3B in H1975 cells treated without (Mock) or with ISD ( $8 \mu \mathrm{g} / \mathrm{ml}$ ) for $3 \mathrm{~h}$. The cells were stained with a mouse anti-human STING and rabbit anti-UBXN3B antibody followed by a secondary antibody conjugated with Alexa Fluor 594/488. The nuclei were stained with DAPI. Objective, $\times 40$. Scale bar, $200 \mu \mathrm{m}$. c A schematic diagram of UBXN3B functional domains. d Co-IP of STING with the truncated forms of UBXN3B. The procedures are similar to b. e Co-IP of UBXN3B with TRIM from HEK293 cells transfected with FLAG-UBXN3B and MycTRIM plasmids using anti-Myc magnetic beads, followed by IB with an anti-Myc (TRIM) and FLAG (UBXN3B) antibody. $\mathbf{f}$ Co-IP of TRIM56 with the truncated forms of UBXN3B from HEK293 cells transfected with FLAG-UBXN3B truncates and Myc-TRIM56 plasmids using anti-FLAG magnetic beads, followed by IB with an anti-Myc (TRIM56) and FLAG (UBXN truncates) antibody. GAPDH is a housekeeping protein control. WCE whole-cell extract. The results are representative of two independent experiments 
further showed that the UAS domain of UBXN3B was required for UBXN3B-STING interaction (Fig. 5c, d; Supplementary Fig. 9 $\mathrm{a}, \mathrm{b})$.

Ubiquitination of STING is essential for its downstream signaling and induction of antiviral immune responses. Several UBXNs including UBXN3B with the UBA-UBX domain are putative adaptor molecules that interact with E3 ligases and their substrates $^{20}$. They likely also determine substrate specificity under different physiological conditions. We hypothesized that UBXN3B might serve as an adaptor that bridges STING and its E3 ligase. Three E3 ligases TRIM32, TRIM56, and AMFR have recently been shown to mediate STING ubiquitination during viral infection ${ }^{7,8,15}$. In particular, TRIM56 mediates both covalent and non-covalent ubiquitination of STING ${ }^{7}$. We first evaluated the ability of each of the E3 ligases to activate STING-dependent IFN-I responses. Results show that overexpression of TRIM56, but not AMFR, dramatically activated and synergized with cGAMP to induce STING-dependent IFN-I, while TRIM32 modestly activated and synergized with cGAMP to induce IFNI (Supplementary Fig. 10). We next determined whether TRIM56 or TRIM32 associated with the STING-UBXN3B complex. UBXN3B co-immunoprecipitated with TRIM56 but not TRIM32 (Fig. 5e). The coil-coiled domain of UBXN3B mediated its interaction with TRIM56 (Fig. 5f; Supplementary Fig. 9c). Very recently, another E3 ligase MUL1 was shown to be essential for STING ubiquitination on K224 and IRF3 activation ${ }^{31}$. We performed co-immunoprecipitation with MUL1 and UBXN3B overexpression in HEK293 cells to see if UBXN3B also regulated MUL1-STING interaction. We however observed no interaction between MUL1 and UBXN3B. These data suggest that UBXN3B specifically regulates STING-TRIM56 interaction. Indeed, HSV-1 infection induced endogenous Sting binding to Trim56 and Ubxn3b in MEFs (Fig. 6a). Furthermore, viral infection-induced Sting-Trim56 binding was almost abrogated in $U b \times n 3 b^{-1-}$ cells but was restored in UBXN3B-reconstituted knockout cells (Fig. 6b). These data overall demonstrate that UBXN3B is critical for HSV-1-induced STING-TRIM56 interaction. We next examined the role of UBXN3B in TRIM56-mediated STING ubiquitination. Overexpression of TRIM56 enhanced endogenous K63-linked ubiquitination of STING in WT cells (HEK293TSTING cell line), but this effect was reduced in $U B X N 3 B^{-1-}$ cells (Fig. 6d) (UBXN3B ${ }^{-l-}$ was generated on HEK293T-STING background (see Fig. 6c). As previously described ${ }^{7}$, K150R mutation decreased STING K63 ubiquitination by TRIM56 (Supplementary Fig. 11a). Trans-complementation of UBXN3B in knockout cells restored normal STING ubiquitination (Fig. 6d).
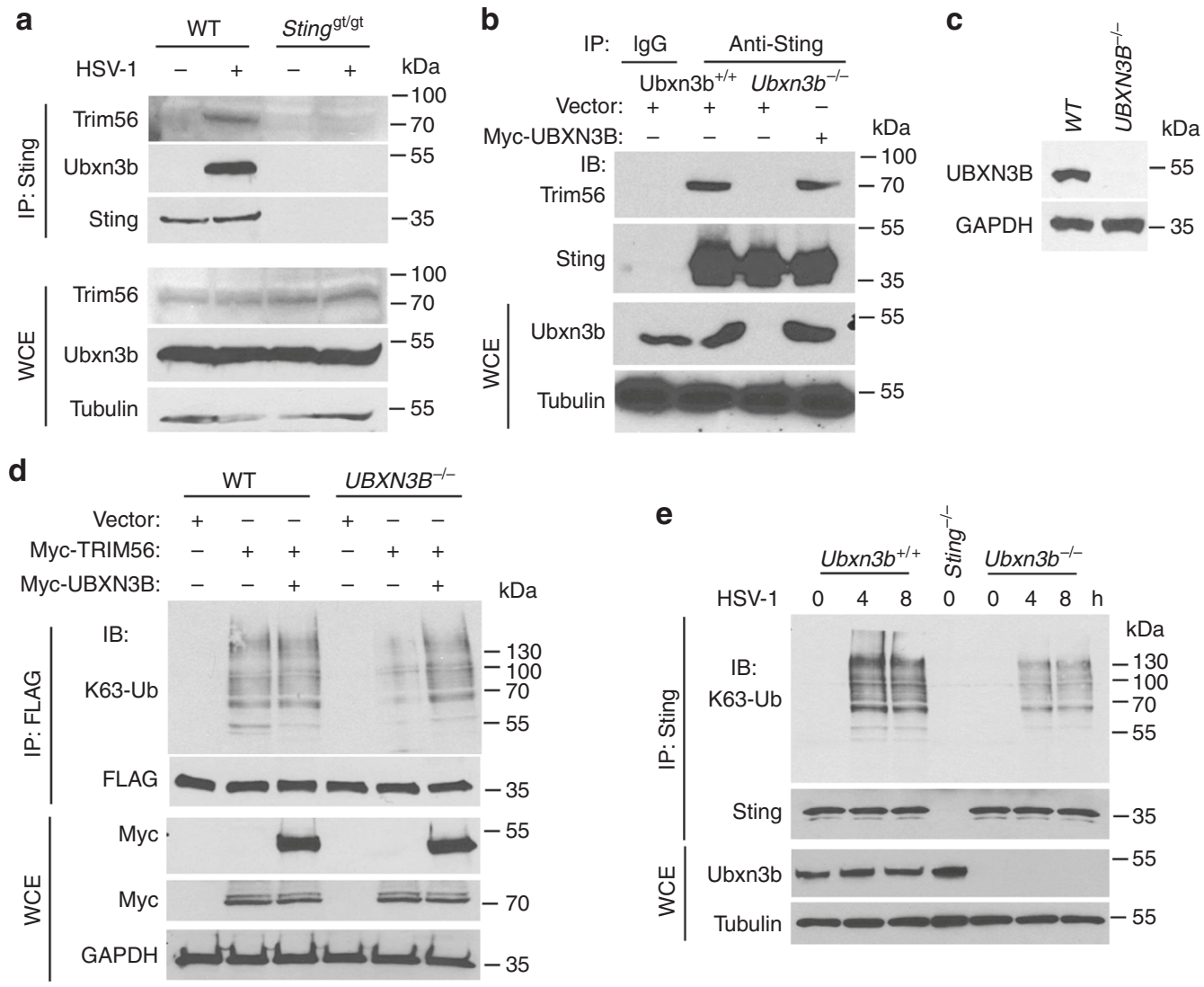

Fig. 6 UBXN3B mediates STING interaction with and ubiquitination by TRIM56. a WT and Stinggt/gt MEFs were infected with or without HSV-1 $(\mathrm{MOI}=0.5)$ for $8 \mathrm{~h}$. Sting was immunoprecipitated $(\mathrm{IP})$ from the MEF lysates using a rabbit anti-Sting antibody. The immunoblots indicate Sting co-immunoprecipitation with Ubxn3b and Trim56 after viral infection. WCE whole-cell extract. b WT and Ubxn3b-/- MEFs were transfected with vector or FLAG-UBXN3B plasmids by electroporation, and then infected with HSV-1 (MOI $=0.5)$ for $8 \mathrm{~h}$. Sting IP was performed as in a. Rabbit IgG was used as a negative control. c The immunoblots show knockout efficacy of UBXN3B in HEK293T-STING cell line. $\mathbf{d}$ WT and UBXN3B ${ }^{-1-}$ cells (parent cell line is HEK293T-FLAG-STING) were transfected with the indicated combinations of plasmids. FLAG-STING was immunoprecipitated (IP) with anti-FLAG magnetic beads. The proteins in IP and WCE were immunoblotted by an anti-K63-linked polyubiquitin, anti-Myc (UBXN3B and TRIM56) and anti-FLAG (STING) antibody, respectively. e Sting was precipitated with a rabbit anti-Sting antibody from cDCs infected without or with $\mathrm{HSV}-1$ (MOI $=10)$ for 4 and $8 \mathrm{~h}$. The Sting ${ }^{-1-}$ cDCs were used as an IP control. The proteins in IP and WCE were immunoblotted with an anti-K63 and Sting antibody, respectively. Tubulin and GAPDH are housekeeping controls. The results are representative of two independent experiments 
To validate the aforementioned overexpression results, we examined endogenous K63 ubiquitination of Sting in cDCs. HSV-1 infection induced K63 ubiquitination of Sting at 4 and $8 \mathrm{~h}$ after infection in $U b \times n 3 b^{+/+}$, but this was obviously impaired in $U b \times n 3 b^{-1-}$ cDCs (Fig. 6e). We recapitulated this in primary human trophoblasts treated with cGAMP (Supplementary Fig. 11b). Of note, co-immunoprecipitation of STING with TBK1 was observed in WT cells, but not in $U B X N 3 B^{-/-}$cells, after cGAMP stimulation (Supplementary Fig. 11b). These data suggest that UBXN3B modulates TRIM56-mediated K63-linked ubiquitination of STING and activation of TBK1.

\section{Discussion}

Being a central molecule of the anti-DNA virus immune signaling pathway, STING is subjected to complex regulation by cellular factors and also many viruses ${ }^{12}$. We here present physiological and biochemical evidence that UBXN3B controls DNA virus infection by regulating STING-mediated antiviral immune responses. First, ectopic expression of UBXN3B potentiates specifically STING-dependent IFN-I induction. Second, Ubxn $3 b^{-/-}$ mice and cells are deficient in IFN-I induction by HSV-1, ISD, and cGAMP that stimulate STING signaling. Third, Ubxn $3 b^{-1-}$ mice are highly susceptible to HSV-1 and VSV infection, but not to EMCV infection, recapitulating the Sting $^{-1-}$ phenotypes. Fourth, UBXN3B interacts with both STING and TRIM56 simultaneously, and HSV-1-induced STING-TRIM56 interaction is compromised in $U B X N 3 B^{-1-}$ cells. Fifth, UBXN3B deficiency leads to decreased K63 ubiquitination and dimerization of STING, and consequent phosphorylation of TBK1 and IRF3. STING translocation out of the ER to the ER-Golgi intermediate compartment, followed by phosphorylation and degradation after HSV-1/ISD/cGAMP stimulation, is also partly blocked in $U B X N 3 B^{-1-}$ cells. Lastly, UBXN3B can be induced by IFN-I, likely to form a positive feedback loop to stimulate antiviral immune responses. All these observations strongly suggest that UBXN3B directly regulate STING signaling. However, STINGmediated immune responses are not completely abolished in $U B X N 3 B^{-1-}$ cells and mice, suggesting that UBXN3Bindependent regulation of STING and IFN-I-inducing pathways exist. Several E3 ligases that may not be directly relevant to UBXN3B have been shown to positively regulate STING signaling $8,15,31$. Moreover, in pDCs TLR9 contributes to induction of the IFN-I responses by HSV-1 ${ }^{32,33}$, which however is not regulated by UBXN3B.

The UBA-UBX-containing UBXN proteins may be adaptors that bridge multiple E3 ligases and a variety of substrates ${ }^{20}$, and likely essential for embryonic development. Indeed, by using different genome editing technologies, we and collaborators independently find that $U b \times n 3 b$-null mutation is embryonically lethal ${ }^{24}$. In agreement with our findings, another UBA-UBX gene, $U B X N 1$, is also essential for embryonic development ${ }^{34}$. The genes essential for embryonic/neonatal stages are generally believed to be essential for adult stage too. For example Gpx4 and p53 is not only essential for embryos/neonates but also for adult mice ${ }^{25,26}$. Surprisingly, adult mice depleted of $U b \times n 3 b$ survive and develop as normally as WT mice. This interesting finding suggests that some of the genes indispensable for embryonic development may become non-essential at later stages of development. Our results provide proof of principle for in-depth study of the physiological functions of essential genes like $U B X N 3 B$ in adult systemic knockout mice.

Of note, Ubxn3b protein expression is still abundant and ubiquitous in adult mice, suggesting that it plays different physiological roles than that at the embryonic stage. Previous studies demonstrated that UBXN3B regulated triglyceride synthesis and lipid droplet turnover in vitro cell cultures ${ }^{22,23}$, and promoted very low-density lipoprotein secretion out of hepatocytes in mice fed with a high-fat $\operatorname{diet}^{24}$. More recently, UBXN3B was shown to regulate de novo sterol biosynthesis by targeting the rate-limiting enzyme of the mevalonate pathway-HMG-CoA reductase to proteasomal degradation ${ }^{35}$. In the current study, we show that under viral infection conditions, UBXN3B bridges the ER protein STING and its cytoplasmic E3 ligase TRIM56 to initiate antiviral immune responses. The multifaceted nature of UBXN3B is likely attributable to its capability as an adaptor to interface different E3 ligases ${ }^{20}$ and substrates under different physiological conditions. The involvement of an adaptor in an E3 ligase action could greatly expand the substrate spectrum of the E3 ligase in response to various physiological and environmental cues.

Following DNA virus infection, STING undergoes four forms of polyubiquitination, K11-linked ${ }^{36}$, K27-linked ${ }^{15}$, K48linked ${ }^{16,17}$, and K63-linked ${ }^{7,8,31}$, resulting in contrasting effects on STING-mediated immune signaling. Particularly, K27-linked and K63-linked polyubiquitination activates STING. In agreement with published data ${ }^{7,8}$, forced expression of K63-E3 ligases TRIM32 and TRIM56 enhanced STING-dependent IFN-I induction and significantly potentiated the cGAMP effects on STING. However, overexpression of a K27-E3 ligase AMFR failed to induce IFN-Is by itself or together with cGAMP, suggesting a different mechanism of AMFR action than TRIM32 and TRIM56. A similar phenomenon has been observed for TRAF3, a K63-E3 ligase critical for TLR-dependent and RLR-dependent IFN-I induction ${ }^{37}$. It is not surprising that redundant K63-E3 ligases are available for STING, since it is crucial for induction of anti-DNA virus immunity. Indeed, recently another K63-E3 ligase MUL1 was shown to ubiquitinate the K224 of STING and specifically regulate IRF3 activation, followed by STING phosphorylation and degradation ${ }^{31}$. Our results demonstrate that UBXN3B regulates TRIM56-mediated ubiquitination of STING. It binds STING via the UAS domain and TRIM56 via the coiled-coil domain, likely serving as an adaptor to bring STING to the vicinity of TRIM56. K63-linked polyubiquitination of STING can be in a covalent form, which is necessary for STING oligomerization and activation of downstream immune responses ${ }^{7}$. Like RIG-I, STING could also be non-covalently modified with and activated by unanchored polyubiquitin chains ${ }^{7,37}$. In this scenario, UBXN3B could play a role by binding and bringing polyubiquitin chains to STING, in light of recent findings that the UBA domain of some UBXNs was able to bind polyubiquitin chains ${ }^{20}$. It is thus possible that UBXN3B could also in part regulate STING ubiquitination catalyzed by other E3 ligases indirectly.

In summary, we present physiological and biochemical evidence that UBXN3B controls DNA virus infection by regulating STING signaling. Our data offer an insight into the previously unappreciated immune regulatory function of UBXN3B and provide proof of methodology for investigating the physiological functions of certain essential genes like $U B X N 3 B$ in adult systemic knockout animals.

\section{Methods}

Mouse models. Mice with the exon 1 of Ubxn3b flanked by two LoxP sites $\left(U b x n 3 b^{\text {flox/flox }}\right)$ were generated via homologous recombination by Dr. Fujimoto at Nagoya University ${ }^{24}$. The homozygous Ubxn $3 b^{\text {flox/flox }}$ were then crossed with homozygous tamoxifen-inducible Cre recombinase-estrogen receptor T2 mice (The Jackson Laboratory, Stock \# 008463) to generate male and female Cre ${ }^{+1-}$ $U b x n 3 b^{\text {flox }}$ littermates, which were mated to produce Cre ${ }^{+/-} U b x n 3 b^{\text {flox/flox }}$, $U b x n 3 b^{\text {flox/flox }}, \mathrm{Cre}^{+l-}$ for experimentation. Genotyping PCR was performed with $\sim 40 \mathrm{ng}$ genomic DNA and Choice Taq Blue Mastermix (Denville Scientific, Cat\# CB4065-8) under the following cycling conditions: $94^{\circ} \mathrm{C}$ for $2 \min \left(94^{\circ} \mathrm{C}\right.$ for 1 min, $60{ }^{\circ} \mathrm{C}$ for $30 \mathrm{~s}, 72^{\circ} \mathrm{C}$ for $\left.30 \mathrm{~s}\right) \times 34$ cycles, $72^{\circ} \mathrm{C}$ for $7 \mathrm{~min}^{24}$. The primers for LoxP were: forward primer, $5^{\prime}$-CCAACCTCTGTGGGTCCTC- $3^{\prime}$ and reverse primer, 5'-GCGATTGGGGATCTGAGTTA-3. This PCR reaction resulted in a product of $187 \mathrm{bp}$ (WT, LoxP negative) and/or $245 \mathrm{bp}$ (mutant, LoxP positive). The 
primers for genotyping Cre were: common-5'AAGGGAGCTGCAGTGGAGTA-3', WT reverse, 5'-CCG AAA ATC TGTGGGAAGTC- ${ }^{\prime}$ ', and mutant reverse, 5' - CG GTTATTCAACTTGCACCA-3'. The PCR reaction resulted in a product of $\sim 300$ bp (WT, Cre negative) and/or 450 bp (mutant, Cre positive). To induce Ubxn3b deletion, weaned mice were treated with $100 \mu \mathrm{l}$ of tamoxifen $(10 \mathrm{mg} / \mathrm{ml}$ in corn oil) (Sigma, \#T5648) by intraperitoneal injection every 2 days for a total duration of 8 days $^{26}$. The treated mice were housed for 1-2 weeks, allowing tamoxifen to be metabolized completely (half-life $\sim 16 \mathrm{~h}$ ). The Sting-deficient strain (Stinggt/gt) was initially developed by Dr. Russell Vance at the University of California, Berkeley ${ }^{38}$, and is now available at the Jackson Laboratory (Stock \# 017537). Sting ${ }^{-1-}$ (null knockout of exons 3-5) (Stock \# 025805) and IFN-I receptor knockout (Ifnar $1^{-1-}$ ) (Stock \# 028288) mice were also from the Jackson Laboratory. We used 8-10-weekold sex-matched (both female and male) mice for all the experiments. All the animal protocols were approved by the Institutional Animal Care and Use Committee at New York Medical College and Yale University adhering to the National institutes of Health recommendations for the care and use of laboratory animals.

Reagents and antibodies. The rabbit anti-GAPDH (D16H11, Cat\# 5174, 1:3000), calreticulin (D3E6, Cat\# 12238, 1:200 for IFA), $\alpha$-Tubulin (Cat\# 2144, 1:3000), UBXN3B (D8H6D, Cat\# 34945, 1:1000 for IB, 1:100 for IFA), TBK1 (D1B4, Cat\# 3504, 1:1000), phospho-TBK1 (D52C2, Cat\# 5483, 1:500), K63-linked polyubiquitin (D7A11, Cat\# 5621,1:500), phospho-IRF3 (4D4G, Cat\# 4947, 1:500), human phospho-STING (Cat\# 85735, 1:500), and STING (D2P2F, Cat\#13647, 1:500) were purchased from Cell Signaling Technology (Danvers, MA, USA). The goat anti-TRIM56 (E-18, Cat\# sc-249085, 1:200) was obtained from Santa Cruz Biotechnology (CA, USA). The goat anti-UBXN3B was obtained from Genway Biotech (Cat\# GWB-5E6F34, 1:500, San Diego, CA, USA). The mouse anti-FLAG (OTI4C5, Cat\# TA50011, 1:2000), Myc (9E10, Cat\# TA150121, 1:1000), and HA (HA.C5, Cat\# TA100012, 1:1000) were obtained from Origene (Rockville, MD, USA). Lipofectamine 2000 was obtained from ThermoFisher (Waltham, MA, USA). 2'3'-cGAMP (Cat\# tlrl-nacga23), IFN stimulatory DNA derived from Listeria monocytogenes genome (ISD, Cat\# tlrl-isdn), polyI:C (1.5-8 kb, Cat\# tlrl-pic), FSL-1 (Pam2CGDPKHPKSF, Cat\# tlll-fsl), CpG DNA (5'-tcgtcgttttcggcgc:gcgccg3', Cat\# tlrl-2395), LPS (Cat\# tlrl-pb5lps), and ssPolyU (Cat\# tlrl-sspu) were products of Invivogen (San Diego, CA, USA). The anti-FLAG M2 magnetic beads (Clone M2, Cat\# M8823) were from Sigma-Aldrich (St. Louis, MO, USA) and antiMyc magnetic beads (Clone 9E10, Cat\# 88842) were from ThermoFisher (Waltham, MA, USA). The mouse anti-human STING (Clone 723505, Cat\# MAB7169, 1:200 for IFA) was available at R\&D Systems (Minneapolis, MN, USA). Recombinant mouse IFN- $\alpha$ (Cat\# 752802) was a product of BioLegend (San Diego, CA, USA). The rabbit anti-HSV-1/HSV-2 serum, which is specific for the ICP's and late structural (virion) antigens, was purchased from Antibodies-online (Cat\# ABIN 285517, 1:500).

Plasmid construction. FLAG-Myc-UBXN2A (Cat\# MR203340, NCBI accession: NM_145441) and FLAG-Myc-UBXN2B (Cat\# MR204884, NCBI accession: NM_026534) on pCMV6-entry vector were purchased from Origene (Rockville, MD, USA). FLAG-UBXN1 was constructed by inserting the human UBXN1 open reading frame (ORF) (NCBI accession: NM_015853.4) into pcDNA3-FLAG/Myc vector $^{39}$. The additional FLAG-UBXNs were a kind gift of Dr. Raymond J. Deshaies at California Institute of Technology, USA ${ }^{20}$. HA-STING and FLAGSTING-K150R were recently reported ${ }^{7,40}$. The truncated forms of human UBXN3B, human AMFR, TRIM32, and TRIM56 were amplified by PCR, cut by restriction enzymes, and inserted into pcDNA3-FLAG/Myc vector.

Cell culture and viruses. EMCV (Cat\# VR129-B), SeV (Cantell strain, Cat\# VR-907), VSV (Indiana strain, Cat\# VR-1238), and HSV-1 (Cat\# VR1493) were purchased from American Type Culture Collection (ATCC) (Manassas, VA, USA) and the multiplicity of infection (MOI) was specified in each figure legend ${ }^{41}$. Green fluorescent protein-tagged herpes simplex virus type I (KOS strain) was provided by Dr. Rongtun Lin at the McGill University ${ }^{28}$. All viruses were propagated in Vero cells.

HEK293T cells (Cat\# CRL-3216), Vero cells (monkey kidney epithelial cells, Cat\# CCL-81), H1975 cells (human lung epithelial cells (Cat\# CR5908), and L929 cells (mouse fibroblast cells, Cat\# CCL-1) were purchased from ATCC (Manassas, VA, USA). TLR4/MD2/CD14-expressing HEK293 cell line was obtained from Invivogen (Cat\# 293-htlr4md2cd14, San Diego, CA, USA). HEK293T/Vero cells and L929/H1975 cells were grown in Dulbecco's modified Eagle's medium or Roswell Park Memorial Institute (RPMI) 1640, respectively (Life Technologies, Grand Island, NY, USA) supplemented with 10\% fetal bovine serum (FBS) and antibiotics/antimycotics. These cell lines are not listed in the database of commonly misidentified cell lines maintained by ICLAC, and have not been authenticated in our hands. They are routinely treated with MycoZAP (Lonza) and tested for mycoplasma contamination in our hands.

Generation of HEK293T-STING cell line. Since HEK293T expresses a very low level of STING, we thus constructed a FLAG-STING-expressing stable cell line. The human STING ORF was subcloned into pcDNA3-FLAG vector, and then transfected into HEK293T cells using Lipofectamine 2000. FLAG-STINGexpressing cells were selected with $200 \mu \mathrm{g} / \mathrm{ml}$ zeocin.

Ubxn3b deletion in primary MEFs. Pregnant $\mathrm{Cre}^{+/-} U b x n 3 b^{\text {flox/flox }}$ females (mated to $\mathrm{Cre}^{+/-} U b \times n 3 b^{\text {flox/flox }}$ male) were euthanized on day 14 of gestation. Embryos were decapitated and eviscerated, and then digested with trypsin for 10 min at $37^{\circ} \mathrm{C}$ rotating. Fibroblasts were filtered through a $100 \mu \mathrm{M}$ filter, cultured in RPMI 1640 medium (Life Technologies, NY, USA), supplemented with 10\% FBS and antibiotics/antimycotics, propagated for two passages, and then frozen ${ }^{41,42}$ $\mathrm{Cre}^{+/-} U b x n 3 b^{\text {flox/flox }}$ MEFs were identified by genotyping. The $\mathrm{Cre}^{+/-}$ $U b x n 3 b^{\text {flox/flox }}$ MEFs were treated with 4-hydroxyl tamoxifen at $0.01 \mathrm{mg} / \mathrm{ml}$ for 3 days to generate $U b x n 3 b^{-l-}$ cells. After induction, the cells were further passaged two times in RPMI medium. Non-treated $\mathrm{Cre}^{+/-} U b x n 3 b^{\text {flox/flox }}$ MEFs were considered $U b x n 3 b^{+/+}$

Differentiation of BMDMs and DCs. BMDMs were differentiated using a published method ${ }^{43}$. Briefly, bone marrow cells were isolated from mock-pretreated

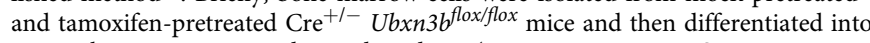
macrophages in L929-conditioned medium (RPMI 1640, 20\% FBS, 30\% L929 culture medium, $1 \times$ antibiotics/antimycotics) in 10 -cm Petri dishes at $37^{\circ} \mathrm{C}, 5 \%$ $\mathrm{CO}_{2}$ for 5 days. The culture medium was replaced by fresh L929-conditioned medium every 2 days. The attached BMDMs were dislodged by pipetting and counted for plating in 12-well or 6-well cell culture plates. Bone marrow-derived $\mathrm{cDCs}$ and pDCs were induced from bone marrow cells with $10 \mathrm{ng} / \mathrm{ml}$ murine GM-CSF and $100 \mathrm{ng} / \mathrm{ml}$ Flt3L (PeproTech), respectively, for 6-8 days ${ }^{44}$. BMDMs/DCs were cultured in RPMI 1640 medium containing $10 \%$ (volume/volume (v/v)) FBS (Invitrogen, Carlsbad, CA, USA), $100 \mathrm{U} / \mathrm{ml}$ penicillin and $100 \mu \mathrm{g} / \mathrm{ml}$ streptomycin (Invitrogen) and maintained at $37^{\circ} \mathrm{C}$ and $5 \% \mathrm{CO}_{2}$ overnight, and then washed once with pre-warmed fresh medium.

Isolation of human trophoblasts. Human trophoblasts were isolated from first trimester or term placenta using published methods ${ }^{45,46}$. Briefly, a piece of placenta was minced with a razor blade and then digested with $0.25 \%$ trypsin and $0.2 \mathrm{mg} / \mathrm{ml}$ DNAse I in Hank's balanced salt solution to release cells. The dispersed cells were passed through a $40 \mu \mathrm{m}$ cell strainer, and trophoblasts were isolated through Percoll gradient $(10-70 \% \mathrm{v} / \mathrm{v})$ centrifugation. Cells were maintained in complete RPMI 1640 medium with 10\% FBS and antibiotics. The IRB of Yale University and New York Medical College approved the study and deemed exemption of human subject study ${ }^{47}$. The study used de-identified leftover specimens which were otherwise discarded. The investigators did not have any contact with the subjects and had no access to the patient information and did not link the results to the subjects. The investigation was irrelevant to clinical care and uses random specimens. The research did not investigate the health/disease status/genetic information of the subjects.

Ligand treatment and viral infection conditions. PRR ligands were transfected into cells with Lipofectamine 2000 using standard procedures. For hard-totransfect cells such as trophoblasts, H1975, MEFs, and DCs, transfection was carried out in cell suspension. Briefly, cells were dislodged by trypsin digestion and pelleted by brief centrifugation. The cell pellet was then suspended in the transfection mix (DNA + Lipofectamine 2000 prepared as above) for $20 \mathrm{~min}$ with intermittent gentle agitation. Pre-warmed RPMI 1640 complete medium was then added and plated for further culture. For viral infection, viruses were allowed to attach and infect cells for $2 \mathrm{~h}$; the cells were then washed with pre-warmed $1 \times$ phosphate-buffered saline (PBS) once and incubated with fresh medium. The MOI and infection time were specified for each experiment in the figure legend.

\section{Generation of gene knockout cell lines with CRISPR-Cas9 technology. Two} target guide sequences (1-GGTCAGTGACCCGGCTGCGA, 2-

TACGTTCCCTGGTAGAAGAC common for both mouse and human UBXN3B) were cloned into lentiCRISPRv2 vector and co-transfected into HEK293T cells with the packaging plasmids pVSVg and psPAX2 ${ }^{48,49}$. The guide RNAs for human STING were: 1-GGTGGAGCACCAGGTAC, 2-GGTACCGGAGAGTGTGCTC). HEK293T-STING cells, trophoblasts, and H1975 cells were then transduced by lentiviral particles. The WT control was lentiCRISPRv2 vector only. The transduced cells were selected with puromycin at $1 \mu \mathrm{g} / \mathrm{ml}$ for trophoblasts, $1 \mu \mathrm{g} / \mathrm{ml}$ for HEK293T cells and $0.5 \mu \mathrm{g} / \mathrm{ml}$ for H1975 cells. Successful knockout clones were confirmed by immunoblotting.

Plaque-forming assay. Quantification of infectious viral particles in tissues/cell culture supernatant was performed on Vero cell monolayer ${ }^{43,50}$. Briefly, $30-100 \mu \mathrm{g}$ (total proteins) of tissue lysates triturated in sterile PBS or serial dilutions of supernatant was applied to confluent Vero cells (6-well plate) at $37^{\circ} \mathrm{C}$ for $2 \mathrm{~h}$. The inoculum was then removed and replaced with $2 \mathrm{ml}$ of DMEM complete medium with $1 \%$ SeaPlaque agarose (Cat\# 50100, Lonza). Plaques were visualized using Neutral red (Sigma-Aldrich) after 3 days of incubation at $37^{\circ} \mathrm{C}, 5 \% \mathrm{CO}_{2}$. 
Mouse infection and disease monitoring. Six-week-old to eight-week-old sex-matched littermates were infected with $1 \times 10^{7}$ plaque-forming units (PFUs) of HSV-1/VSV intravenously or $200 \mathrm{PFU}$ of EMCV intraperitoneally. Morbidity and mortality was monitored twice a day. Neurological symptoms were assessed using an arbitrary scoring criteria from 1 to 5 (where 1 indicated ruffled fur and hunched posture but can easily be made to move around; 2 indicated a hunched posture and slow to move; 3 indicated a hunched posture, some movement, and labored breathing; 4 indicated a hunched posture, labored breathing, and little or no movement; and 5 indicated moribund or dead $)^{51}$

Reverse transcription and qPCR. A few grams of animal tissues, $25 \mu$ l of whole blood, and up to $1 \times 10^{6}$ culture cells were collected in $350 \mu$ l of RLT buffer (QIAGEN RNeasy Mini Kit). Soft tissues were homogenized using an electric pellet pestle (Kimble Chase LLC, USA). RNA was extracted following the QIAGEN RNeasy manufacturer's instructions. Reverse transcription of RNA into complementary DNA (cDNA) was performed using the BIO-RAD iScript ${ }^{\text {th }} \mathrm{cDNA}$ Synthesis Kit. Quantitative PCR (qPCR) was performed with gene-specific primers and 6FAM-TAMRA (6-carboxyfluorescein- $N, N, N, N$-tetramethyl-6-carboxyrhodamine) probes or SYBR Green. Results were calculated using the $-\Delta \Delta \mathrm{Ct}$ method and a housekeeping gene as an internal control. The qPCR primers and probes for immune genes were reported in our previous studies 42,52 . The primer pair for mouse Ubxn3b was 5'-GAGAAATATGGGAGGGCACA- ${ }^{\prime}$ and $5^{\prime}$-AGAA CAAGCCCAGAAAAGCA- $3^{\prime}$. The primers for HSV-1 were: $5^{\prime}$-AGCCTGTACC

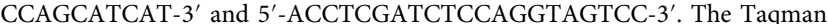
gene expression assays for Ifit1 (Mm00515153_m1), Oas1a (Mm00836412_m1), Isg15 (Mm01705338 s1), IFNB1 (Hs01077958 s1), and TNFA (Hs00174128 m1) were obtained from ThermoFisher Scientific. The housekeeping gene controls were beta actin $(A C T B)$, peptidylprolyl isomerase A (PPIA), and hydroxymethylbilane synthase $(H M B S)$. A previous study demonstrated that $H M B S$ was the least unaffected housekeeping gene during HSV-1 infection; PPIA was most stable for other viral infections ${ }^{53}$. Human HMBS primers are: 5' -ACGGCTCAGATAGCA TACAAGAG- ${ }^{\prime}$ and $5^{\prime}$-GTTACGAGCAGTGATGCCTACC- ${ }^{\prime}$; mouse Hmbs: 5'-GTGCCTACCATACTACCTCCTG-3' and 5' -ACTCTCCTCAGAGAGCTGGT TC-3'; human PPIA: $5^{\prime}$-GGCAAATGCTGGACCCAACACA-3' and 5'-TGCTGG TCTTGCCATTCCTGGA-3'; mouse Ppia: 5'-CATACAGGTCCTGGCATCTTG TC- $3^{\prime}$ and $5^{\prime}$-AGACCACATGCTTGCCATCCAG- ${ }^{\prime}$. These primer sequences were available at Origene Technologies (Rockville, MD, USA).

ELISA. A commercial ELISA (enzyme-linked immunosorbent assay) Kit was used to measure the levels of IFN- $\alpha$ (Cat\# 42120) and IFN- $\beta$ (Cat\# 42400, PBL Assay Sciences, Piscataway Township, NJ, USA) proteins in either cell culture supernatants or mouse sera.

Immunoblotting. Standard sodium dodecyl sulfate-polyacrylamide gel electrophoresis, Western blotting, and an enhanced chemiluminescent (ECL) substrate (ThermoFisher, Cat\# 32106) was applied except for STING dimerization assay where no reducing agent was applied to the samples. In some cases, such as K63-Ub, an ultra-sensitive ECL substrate for low-femtogram-level detection (ThermoFisher, Cat\# 34095) was used.

Co-immunoprecipitation. HEK293T cells were transfected with expression plasmids using Lipofectamine 2000. Whole-cell extracts were prepared from transfected cells in lysis buffer $(150 \mathrm{mM} \mathrm{NaCl}, 50 \mathrm{mM}$ Tris, $\mathrm{pH} 7.5,1 \mathrm{mM}$ EDTA, $0.5 \%$ NP40, $10 \%$ glycerol) and were incubated with $50 \mu$ of anti-FLAG magnetic beads for $2 \mathrm{~h}$ at $4{ }^{\circ} \mathrm{C}$. Co-immunoprecipitation was performed according to the manufacturer's instructions (anti-Flag magnetic beads, Sigma-Aldrich)

Co-immunoprecipitation of endogenous proteins. A total of $1 \times 10^{7}$ cells were lysed in $2 \mathrm{ml}$ of lysis buffer ( $150 \mathrm{mM} \mathrm{NaCl}, 50 \mathrm{mM}$ Tris, pH 7.5, $1 \mathrm{mM}$ EDTA, $0.5 \%$ NP40, 10\% glycerol, protease inhibitor cocktail). Lysates were cleared by centrifugation at $6000 \times g$ for $10 \mathrm{~min}$ at $4^{\circ} \mathrm{C}$. For UBXN3B trans-complementation, MEFs were transfected with $5 \mu \mathrm{g}$ vector or FLAG-UBXN3B per $1 \times 10^{6}$ cells respectively, by electroporation (Lonza, Cat\# DCR1003) $20 \mathrm{~h}$ before HSV-1 infection. Two micrograms of rabbit IgG or $50 \mu \mathrm{l}$ of anti-STING/per sample (Cell Signaling Technology, Cat\# 13647) was cross-linked to $50 \mu \mathrm{l}$ of protein A/G agarose beads (ThermoFisher, Cat\# 20421) with dimethyl pimelimidate (ThermoFisher, Cat\# 21666). The cleared lysates were incubated with the agarose beads with gentle agitation at $4{ }^{\circ} \mathrm{C}$ overnight. The beads were washed five times in icecold wash buffer (150 mM NaCl, $50 \mathrm{mM}$ Tris, pH 7.5, 1 mM EDTA, 0.5\% NP40), and bound proteins were eluted by boiling for $3 \mathrm{~min}$ in SDS sample lysis buffer.

Dual luciferase reporter assays. Seventy percent confluent HEK293T cells were transfected with $50 \mathrm{ng}$ of pRL-TK reporter (herpes simplex virus thymidine kinase promoter-driven renilla luciferase; internal control), $100 \mathrm{ng}$ of pGL3-ISRE luciferase reporter (firefly luciferase; experimental reporter) plasmid, and other expressing plasmids as appropriate. Twenty-four hours after transfection, luciferase activity was measured using a Promega Dual Glow Kit (Cat\# E2980) according to the manufacturer's instructions.
Immunofluorescent microscopy. Cells were fixed with $4 \%$ paraformaldehyde. The cells were sequentially permeabilized with $0.5 \%$ Triton X-100, blocked with $2 \%$ goat serum, incubated with primary antibody (rabbit anti-UBXN3B, 1:100, Cell Signaling (Cat\# 34945, Clone D8H6D); mouse anti-STING, 1:100, R\&D Systems (Clone 723505, Cat\# MAB7169); and rabbit anti-calreticulin, 1:200, Cell Signaling (Clone D3E6, Cat\# 12238)) at $4{ }^{\circ} \mathrm{C}$ overnight, washed briefly, and then incubated with Alexa Fluor 488/594-conjugated goat anti-rabbit/mouse IgGs (1:200, Life Technologies, Cat\# A11070) for $1 \mathrm{~h}$ at room temperature. Nuclei were counterstained with DAPI (4',6-diamidino-2-phenylindole). Images were acquired using a Zeiss Axiovert-200 fluorescence microscope (objective, $\times 40$ ).

Graphing and statistics. The sample size chosen for our animal experiments in this study was estimated based on our prior experience of performing similar sets of experiments and power analysis calculations (http://isogenic.info/html/ power_analysis.html). All animal results were included and no method of randomization was applied. For all the bar graphs, data were expressed as mean \pm s.e.m. Prism 7 software (GraphPad Software) was used for survival curves, charts, and statistical analyses. Survival curves were analyzed using a log-rank (Mantel-Cox) test. For in vitro results, a standard two-tailed unpaired Student's $t$ test was used. For animal studies, an unpaired two-tailed non-parametric/parametric Mann-Whitney $U$ test was applied to statistical analysis. $P$ values $\leq 0.05$ were considered significant. The sample sizes (biological replicates), specific statistical tests used, and the main effects of our statistical analyses for each experiment were detailed in each figure legend.

Data availability. The datasets generated during and/or analyzed during the current study are available from the corresponding author upon request.

Received: 22 August 2017 Accepted: 23 May 2018

Published online: 13 June 2018

\section{References}

1. Sun, L., Wu, J., Du, F., Chen, X. \& Chen, Z. J. Cyclic GMP-AMP synthase is a cytosolic DNA sensor that activates the type I interferon pathway. Science 339, 786-791 (2013).

2. $\mathrm{Wu}$, J. et al. Cyclic GMP-AMP is an endogenous second messenger in innate immune signaling by cytosolic DNA. Science 339, 826-830 (2013).

3. Ablasser, A. et al. cGAS produces a $2^{\prime}-5^{\prime}$-linked cyclic dinucleotide second messenger that activates STING. Nature 498, 380-384 (2013).

4. Zhong, B. et al. The adaptor protein MITA links virus-sensing receptors to IRF3 transcription factor activation. Immunity 29, 538-550 (2008).

5. Ishikawa, H. \& Barber, G. N. STING is an endoplasmic reticulum adaptor that facilitates innate immune signalling. Nature 455, 674-678 (2008).

6. Ishikawa, H., Ma, Z. \& Barber, G. N. STING regulates intracellular DNAmediated, type I interferon-dependent innate immunity. Nature 461, 788-792 (2009)

7. Tsuchida, T. et al. The ubiquitin ligase TRIM56 regulates innate immune responses to intracellular double-stranded DNA. Immunity 33, 765-776 (2010).

8. Zhang, J., Hu, M. M., Wang, Y. Y. \& Shu, H. B. TRIM32 protein modulates type I interferon induction and cellular antiviral response by targeting MITA/STING protein for K63-linked ubiquitination. J. Biol. Chem. 287, 28646-28655 (2012).

9. Saitoh, T. et al. Atg9a controls dsDNA-driven dynamic translocation of STING and the innate immune response. Proc. Natl. Acad. Sci. USA 106, 20842-20846 (2009).

10. Dobbs, N. et al. STING activation by translocation from the ER is associated with infection and autoinflammatory disease. Cell Host Microbe 18, 157-168 (2015).

11. Tanaka, Y. \& Chen, Z. J. STING specifies IRF3 phosphorylation by TBK1 in the cytosolic DNA signaling pathway. Sci. Signal. 5, ra20 (2012).

12. Chen, Q., Sun, L. \& Chen, Z. J. Regulation and function of the cGAS-STING pathway of cytosolic DNA sensing. Nat. Immunol. 17, 1142-1149 (2016).

13. Liu, S. et al. Phosphorylation of innate immune adaptor proteins MAVS, STING, and TRIF induces IRF3 activation. Science 347, aaa2630 (2015).

14. Guo, H. et al. NLRX1 sequesters STING to negatively regulate the interferon response, thereby facilitating the replication of HIV-1 and DNA viruses. Cell Host Microbe 19, 515-528 (2016).

15. Wang, Q. et al. The E3 ubiquitin ligase AMFR and INSIG1 bridge the activation of TBK1 kinase by modifying the adaptor STING. Immunity 41, 919-933 (2014)

16. Zhong, B. et al. The ubiquitin ligase RNF5 regulates antiviral responses by mediating degradation of the adaptor protein MITA. Immunity 30, 397-407 (2009). 
17. Wang, Y. et al. TRIM30alpha is a negative-feedback regulator of the intracellular DNA and DNA virus-triggered response by targeting STING. PLoS Pathog. 11, e1005012 (2015).

18. Schuberth, C. \& Buchberger, A. UBX domain proteins: major regulators of the AAA ATPase Cdc48/p97. Cell. Mol. Life Sci. 65, 2360-2371 (2008).

19. Kondo, H. et al. p47 is a cofactor for p97-mediated membrane fusion. Nature 388, 75-78 (1997).

20. Alexandru, G. et al. UBXD7 binds multiple ubiquitin ligases and implicates p97 in HIF1alpha turnover. Cell 134, 804-816 (2008)

21. Gao, D. et al. Cyclic GMP-AMP synthase is an innate immune sensor of HIV and other retroviruses. Science 341, 903-906 (2013).

22. Lee, J. N. et al. Identification of Ubxd8 protein as a sensor for unsaturated fatty acids and regulator of triglyceride synthesis. Proc. Natl. Acad. Sci. USA 107, 21424-21429 (2010).

23. Olzmann, J. A., Richter, C. M. \& Kopito, R. R. Spatial regulation of UBXD8 and p97/VCP controls ATGL-mediated lipid droplet turnover. Proc. Natl. Acad. Sci. USA 110, 1345-1350 (2013).

24. Imai, N. et al. Hepatocyte-specific depletion of UBXD8 induces periportal steatosis in mice fed a high-fat diet. PLoS ONE 10, e0127114 (2015).

25. Yoo, S. E. et al. Gpx4 ablation in adult mice results in a lethal phenotype accompanied by neuronal loss in brain. Free Radic. Biol. Med. 52, 1820-1827 (2012).

26. Ventura, A. et al. Restoration of p53 function leads to tumour regression in vivo. Nature 445, 661-665 (2007)

27. Graham, C. H. et al. Establishment and characterization of first trimester human trophoblast cells with extended lifespan. Exp. Cell Res. 206, 204-211 (1993).

28. Liu, Y. et al. RIG-I-mediated STING upregulation restricts herpes simplex virus 1 infection. J. Virol. 90, 9406-9419 (2016).

29. Konno, H., Konno, K. \& Barber, G. N. Cyclic dinucleotides trigger ULK1 (ATG1) phosphorylation of STING to prevent sustained innate immune signaling. Cell 155, 688-698 (2013).

30. Rueckert, C. et al. Cyclic dinucleotides modulate induced type I IFN responses in innate immune cells by degradation of STING. FASEB J. 31, 3107-3115 (2017).

31. Ni, G., Konno, H. \& Barber, G. N. Ubiquitination of STING at lysine 224 controls IRF3 activation. Sci. Immunol. 2, doi: 10.1126/sciimmunol.aah7119 (2017).

32. Krug, A. et al. Herpes simplex virus type 1 activates murine natural interferonproducing cells through toll-like receptor 9. Blood 103, 1433-1437 (2004).

33. Hoelzer, K., Shackelton, L. A. \& Parrish, C. R. Presence and role of cytosine methylation in DNA viruses of animals. Nucleic Acids Res. 36, 2825-2837 (2008).

34. $\mathrm{Hu}, \mathrm{Y}$. et al. Multiple UBXN family members inhibit retrovirus and lentivirus production and canonical NFkappaBeta signaling by stabilizing IkappaBalpha. PLoS Pathog. 13, e1006187 (2017).

35. Loregger, A. et al. Haploid mammalian genetic screen identifies UBXD8 as a key determinant of HMGCR degradation and cholesterol biosynthesis. Arterioscler. Thromb. Vasc. Biol. 37, 2064-2074 (2017).

36. Qin, Y. et al. RNF26 temporally regulates virus-triggered type I interferon induction by two distinct mechanisms. PLoS Pathog. 10, e1004358 (2014).

37. Zeng, W. et al. Reconstitution of the RIG-I pathway reveals a signaling role of unanchored polyubiquitin chains in innate immunity. Cell 141, 315-330 (2010).

38. Sauer, J. D. et al. The $N$-ethyl- $N$-nitrosourea-induced Goldenticket mouse mutant reveals an essential function of Sting in the in vivo interferon response to Listeria monocytogenes and cyclic dinucleotides. Infect. Immun. 79, 688-694 (2011).

39. Wang, P. et al. UBXN1 interferes with Rig-I-like receptor-mediated antiviral immune response by targeting MAVS. Cell Rep. 3, 1057-1070 (2013).

40. You, F. et al. ELF4 is critical for induction of type I interferon and the host antiviral response. Nat. Immunol. 14, 1237-1246 (2013).

41. Wang, P. et al. Nlrp6 regulates intestinal antiviral innate immunity. Science 350, 826-830 (2015)

42. Wang, P. et al. Caspase-12 controls West Nile virus infection via the viral RNA receptor RIG-I. Nat. Immunol. 11, 912-919 (2010).

43. Wang, P. et al. Matrix metalloproteinase 9 facilitates West Nile virus entry into the brain. J. Virol. 82, 8978-8985 (2008).
44. Li, X. D. et al. Pivotal roles of cGAS-cGAMP signaling in antiviral defense and immune adjuvant effects. Science 341, 1390-1394 (2013).

45. Abrahams, V. M., Straszewski-Chavez, S. L., Guller, S. \& Mor, G. First trimester trophoblast cells secrete Fas ligand which induces immune cell apoptosis. Mol. Hum. Reprod. 10, 55-63 (2004).

46. Aagaard, K. M. et al. Primary human placental trophoblasts are permissive for Zika virus (ZIKV) replication. Sci. Rep. 7, 41389 (2017).

47. Jurado, K. A. et al. Zika virus productively infects primary human placentaspecific macrophages. JCI Insight 1, doi:10.1172/jci.insight.88461 (2016).

48. Sanjana, N. E., Shalem, O. \& Zhang, F. Improved vectors and genome-wide libraries for CRISPR screening. Nat. Methods 11, 783-784 (2014).

49. Shalem, O. et al. Genome-scale CRISPR-Cas9 knockout screening in human cells. Science 343, 84-87 (2014).

50. Wang, P. Exploration of West Nile virus infection in mouse models. Methods Mol. Biol. 1435, 71-81 (2016).

51. Karaba, A. H., Kopp, S. J. \& Longnecker, R. Herpesvirus entry mediator and nectin-1 mediate herpes simplex virus 1 infection of the murine cornea. $J$. Virol. 85, 10041-10047 (2011).

52. Wang, P. et al. IL-22 signaling contributes to West Nile encephalitis pathogenesis. PLoS ONE 7, e44153 (2012)

53. Watson, S. et al. Determination of suitable housekeeping genes for normalisation of quantitative real time PCR analysis of cells infected with human immunodeficiency virus and herpes viruses. Virol. J. 4, 130 (2007).

\section{Acknowledgements}

This work was supported by a National Institutes of Health grant R01AI132526 to P.W. U19 AI089992 and R01 AI127865 to E.F. E.F. is also an investigator of the Howard Hughes Medical Institute.

\section{Author contributions}

L.Y. performed the majority of the experimental procedures. G.Y., L.W., S.C., J.M., H.K., T.G., and D.G.M. contributed to some of the experiments. T.F., G.C., F.Y., R.L., and E.F. contributed to data analysis and/or provided technical support. L.Y. and P.W. conceived and designed; P.W. oversaw the study. L.Y. and P.W. wrote the paper and all the authors reviewed and/or modified the manuscript.

\section{Additional information}

Supplementary Information accompanies this paper at https://doi.org/10.1038/s41467 018-04759-8.

Competing interests: The authors declare no competing interests.

Reprints and permission information is available online at http://npg.nature.com/ reprintsandpermissions/

Publisher's note: Springer Nature remains neutral with regard to jurisdictional claims in published maps and institutional affiliations.

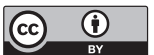

Open Access This article is licensed under a Creative Commons Attribution 4.0 International License, which permits use, sharing, adaptation, distribution and reproduction in any medium or format, as long as you give appropriate credit to the original author(s) and the source, provide a link to the Creative Commons license, and indicate if changes were made. The images or other third party material in this article are included in the article's Creative Commons license, unless indicated otherwise in a credit line to the material. If material is not included in the article's Creative Commons license and your intended use is not permitted by statutory regulation or exceeds the permitted use, you will need to obtain permission directly from the copyright holder. To view a copy of this license, visit http://creativecommons.org/ licenses/by/4.0/.

(c) The Author(s) 2018 